Supporting Information for:

\title{
Molecular Wire Behavior in $\pi$-Stacked Donor-Bridge-Acceptor Tertiary Arylureas
}

Tarek A. Zeidan, Qiang Wang, Torsten Fiebig* and Frederick D. Lewis*

Email: lewis@chem.northwestern.edu,fiebig@bc.edu

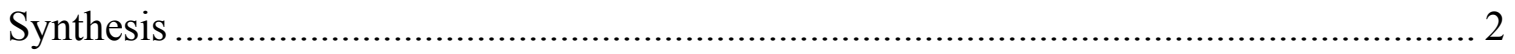

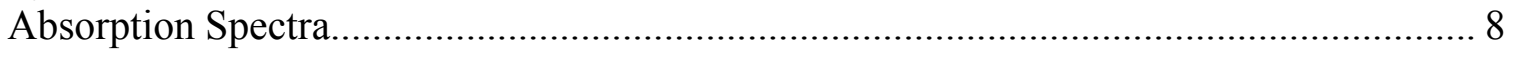

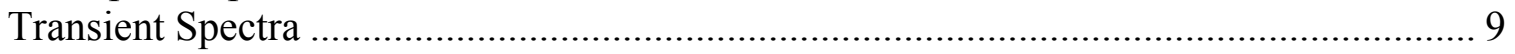

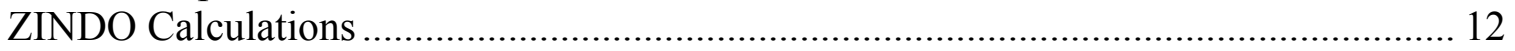

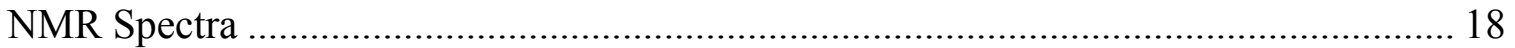

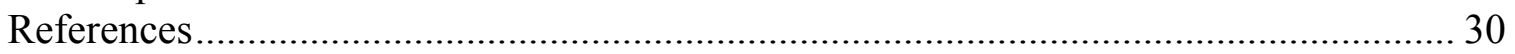




\section{Synthesis}

Materials. Phosgene (20\% solution in toluene), triethylamine, 1-aminopyrene, Nmethylaniline, $p$-phenylenediamine, sodium hydride and methyl iodide are commercially available and used as received. All solvents used for spectroscopy were spectrograde. The preparation of N,N'-diemthyl-p-phenylenediamine dihydrochloride has been previously described. ${ }^{1}$
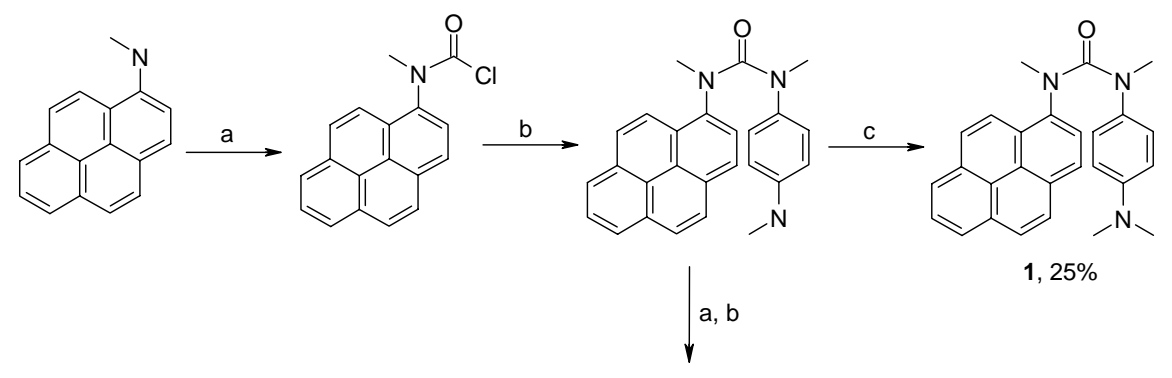

$1,25 \%$
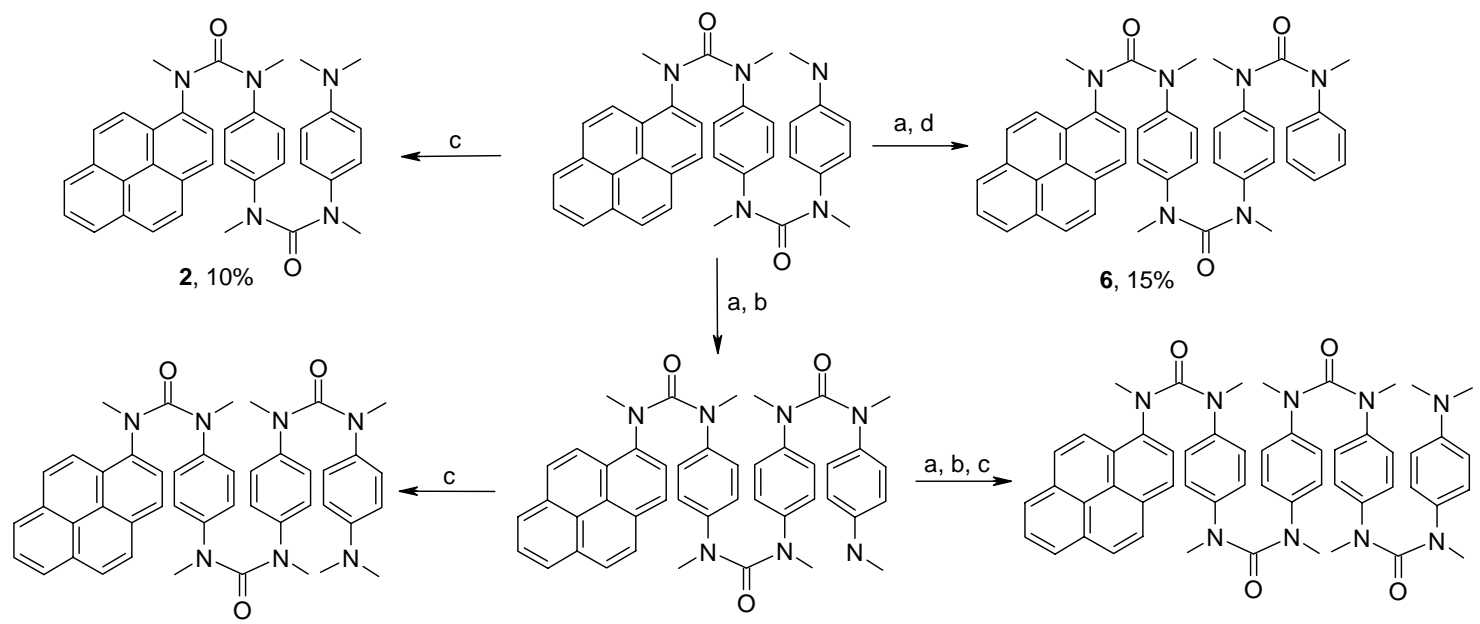

$3,2 \%$

$\downarrow a, b, c$

4, $1.5 \%$

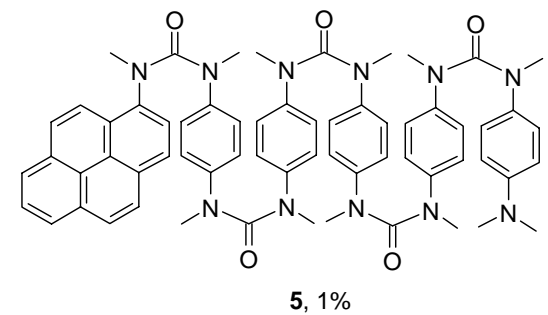

Reagents: a) Phosgene, $\mathrm{Et}_{3} \mathrm{~N}$, toluene, reflux. b) $\mathrm{N}, \mathrm{N}^{\prime}$-dimethyl-phenylenediamine Dihydrochlorid, $\mathrm{Et}_{3} \mathrm{~N}$, toluene, reflux. c) $\mathrm{NaH}, \mathrm{Mel}, \mathrm{DMF}$ d) $\mathrm{N}$-methylaniline, $\mathrm{Et}_{3} \mathrm{~N}$, toluene, reflux 


\section{GENERAL SYNTHETIC PROCEDURES}

Reaction of Secondary Amine with Phosgene. The secondary amine was dissolved in dry toluene with triethylamine. The mixture was added dropwise to a stirring solution of 2.5 equiv. of phosgene $(20 \%$ in toluene) under nitrogen over $10 \mathrm{~min}$. The solution was refluxed for 2 hrs. The reaction mixture was evaporated to dryness and the resulting residue was dissolved in $\mathrm{CH}_{2} \mathrm{Cl}_{2}$. The organic solution was washed with $\mathrm{H}_{2} \mathrm{O}$ and dried over anhydrous $\mathrm{Na}_{2} \mathrm{SO}_{4}$. The solvent was removed under vacuo and the residue was used for the following step without further purification.

\section{Addition of $N, N^{\prime}$-Diemthyl-p-phenylenediamine Dihydrochloride or $\mathrm{N}$ -}

Methylaniline. The acyl chloride was dissolved in dry toluene with triethylamine. The mixture was added slowly to a refluxing solution of $\mathrm{N}, \mathrm{N}^{\prime}$-diemthylphenylenediamine dihydrochloride (or N-methylaniline) with triethylamine in toluene over $1 \mathrm{hr}$. The reaction was refluxed for another $10 \mathrm{~min}$. The reaction mixture was evaporated to dryness and the resulting residue was dissolved in $\mathrm{CH}_{2} \mathrm{Cl}_{2}$. The organic solution was washed with $\mathrm{H}_{2} \mathrm{O}$ and dried over anhydrous $\mathrm{Na}_{2} \mathrm{SO}_{4}$. The solvent was removed under vacuo and the residue was used for the following step without further purification.

Methylation. The urea was dissolved in dry DMF under nitrogen. $\mathrm{NaH}$ was washed with hexanes before usage. A suspension of $\mathrm{NaH}$ in dry DMF was added in portions to the urea solution followed with addition of MeI with every portion over $20 \mathrm{~min}$. The mixture was left stirring under nitrogen for another $3 \mathrm{hrs}$. Water was added to the reaction mixture to precipitate the product. The off-white solid was filtered, dissolved in $\mathrm{CH}_{2} \mathrm{Cl}_{2}$ and dried over anhydrous $\mathrm{Na}_{2} \mathrm{SO}_{4}$. The solvent was removed under vacuo. 


\section{EXPERIMENTAL SECTION}

General. ${ }^{1} \mathrm{H}$ NMR spectra were measured at 400 or $500 \mathrm{MHz}$ in $\mathrm{CDCl}_{3}$ solution with TMS as internal standard. Chemical shifts $(\delta)$ are quoted in parts per million. $J$ values are given in hertz. UV-VIS spectra were measured on a diode array spectrometer using a 1 $\mathrm{cm}$ path length quartz cell. Emission spectra are uncorrected, and the estimated error for the quantum yields is $\pm 10 \%$.

Ground state structures were optimized with the AM1 method implemented in MOPAC as implemented in CAChe $6 \cdot 1 \cdot 10 .{ }^{2}$ Electronic structure calculations were performed on a PC with the ZINDO Hamiltonian (26 occupied and 26 unoccupied orbitals) as implemented in CAChe 6.1.10. All data-fitting procedures were carried out by using Origin (version 6.1). ${ }^{3}$

Materials. Phosgene ( $20 \%$ solution in toluene), triethylamine, 1-aminopyrene, $p$ phenylenediamine, sodium hydride and methyl iodide are commercially available and used as received. All solvents used for spectroscopy were spectrograde. The preparation of $N, N$ '-diemthyl-p-phenylenediamine dihydrochloride has been previously described. ${ }^{1}$

1-(4-Dimethylamino-phenyl)-1,3-dimethyl-3-pyren-1-yl-urea (1). 25\% overall-yield. mp 141-143 ${ }^{\circ} \mathrm{C} ;{ }^{1} \mathrm{H}$ NMR $\left(\mathrm{CDCl}_{3}, 500 \mathrm{MHz}\right): \delta 8.10(\mathrm{dd}, J=7.5,2.0 \mathrm{~Hz}, 2 \mathrm{H}), 7.99-7.83$ (m, 6H), $7.45(\mathrm{~d}, J=8.0 \mathrm{~Hz}, 1 \mathrm{H}), 6.15(\mathrm{~d}, J=8.5 \mathrm{~Hz}, 2 \mathrm{H}), 5.64(\mathrm{~d}, J=9.0 \mathrm{~Hz}, 2 \mathrm{H}), 3.43$ (s, 3H), 3.14 (s, 3H), 2.09 (s, 6H); ${ }^{13} \mathrm{C} \mathrm{NMR}\left(\mathrm{CDCl}_{3}, 100 \mathrm{MHz}\right): \delta$ 162.7, 148.1, 140.1, 134.1, 131.2, 131.1, 129.9, 127.3, 127.2, 127.1, 126.1, 125.9, 125.4, 125.2, 112.6, 40.6, 40.4, 40.3. HRMS (EI) calcd for C27H25O1N3: 407.1992; found 407.1993.

\section{1-(4-Dimethylamino-phenyl)-3-[4-(1,3-dimethyl-3-pyren-1-yl-ureido)-phenyl]-1,3-}

dimethyl-urea (2). $10 \%$ overall-yield. mp $186-190{ }^{\circ} \mathrm{C} ;{ }^{1} \mathrm{H} \mathrm{NMR}\left(\mathrm{CDCl}_{3}, 400 \mathrm{MHz}\right): \delta$ 
8.12-7.82 (m, 7H), $7.75(\mathrm{~d}, \mathrm{~J}=8.8,1 \mathrm{H}), 7.45(\mathrm{~d}, J=8.8 \mathrm{~Hz}, 1 \mathrm{H}), 6.26(\mathrm{~d}, J=8.8 \mathrm{~Hz}$, 2H), $6.17(\mathrm{~d}, J=8.8 \mathrm{~Hz}, 2 \mathrm{H}), 6.07$ (d, $J=8.0 \mathrm{~Hz}, 2 \mathrm{H}), 5.80(\mathrm{~d}, J=8.8 \mathrm{~Hz}, 2 \mathrm{H}), 3.42(\mathrm{~s}$, 3H), 3.10 (s, 3H), 2.86 (s, 3H), 2.73 (s, 6H); ${ }^{13} \mathrm{C} \mathrm{NMR}\left(\mathrm{CDCl}_{3}, 100 \mathrm{MHz}\right): \delta$ 162.1, $161.3,148.3,142.8,141.5,139.4,135.0,131.1,130.9,129.9,127.5,127.2,126.4,126.2$, $125.9,125.6,125.4,125.2,125.0,124.5,122.7,112.5,40.9,40.4,40.1,39.8,38.5$. HRMS (EI) calcd for C36H35O2N5: 569.2785; found 569.2789.

Urea 3. $2 \%$ overall-yield. mp 188-193 ${ }^{\circ} \mathrm{C} ;{ }^{1} \mathrm{H} \mathrm{NMR}\left(\mathrm{CDCl}_{3}, 500 \mathrm{MHz}\right): \delta 8.10(\mathrm{dd}, J$ $=7.0 \mathrm{~Hz}, 2 \mathrm{H}), 7.96(\mathrm{~d}, J=8.0 \mathrm{~Hz}, 2 \mathrm{H}), 7.91-7.83(\mathrm{~m}, 3 \mathrm{H}), 7.74(\mathrm{~d}, J=9.0 \mathrm{~Hz}, 1 \mathrm{H}), 7.45$ (d, $J=8.0 \mathrm{~Hz}, 1 \mathrm{H}), 6.46(\mathrm{~d}, J=9.0 \mathrm{~Hz}, 1 \mathrm{H}), 6.43(\mathrm{~d}, J=8.5 \mathrm{~Hz}, 1 \mathrm{H}), 6.30(\mathrm{~m}, 2 \mathrm{H}), 6.17-$ $6.06(\mathrm{~m}, 6 \mathrm{H}), 6.80(\mathrm{~d}, J=8.5 \mathrm{~Hz}, 2 \mathrm{H}) 3.40(\mathrm{~s}, 3 \mathrm{H}), 3.03(\mathrm{~s}, 6 \mathrm{H}), 2.91(\mathrm{~s}, 3 \mathrm{H}), 2.80(\mathrm{~s}$, 3H), 2.76 (s, 3H), 2.66 (s, 3H), 1.88 (s, 3H); ${ }^{13} \mathrm{C} \mathrm{NMR}\left(\mathrm{CDCl}_{3}, 100 \mathrm{MHz}\right): \delta$ 162.7, $142.9,142,4,142.0,139.3 .1,131.1,131.0,130.8,129.8,129.0,127.5,127.3,127.1$, $127.0,126.6,126.4,126.1,125.8,125.6,125.4,125.0,124.4,122.6,112.3,40.4,40.3$, 40.2, 39.9, 39.6, 39.0, 38.7, 38.4. HRMS (EI) calcd for C45H45O3N7: 731.3578; found 731.3579 .

Urea 4. $1.5 \%$ overall-yield. mp 190-194 ${ }^{\circ} \mathrm{C} ;{ }^{1} \mathrm{H}$ NMR $\left(\mathrm{CDCl}_{3}, 500 \mathrm{MHz}\right): \delta 8.11-7.83$ (m, 7H), $7.73(\mathrm{~d}, J=9.0 \mathrm{~Hz}, 1 \mathrm{H}), 7.45(\mathrm{~d}, J=8.0 \mathrm{~Hz}, 1 \mathrm{H}), 6.53(\mathrm{~d}, J=9.0 \mathrm{~Hz}, 1 \mathrm{H}), 6.45$ (d, $J=8.5 \mathrm{~Hz}, 1 \mathrm{H}), 6.43-6.09$ (m, 12H), 6.77 (d, $J=8.0 \mathrm{~Hz}, 2 \mathrm{H}), 3.39$ (s, 3H), 3.05 (m, 6H), 3.01 (s, 3H), 2.93 (s, 3H), $2.90(\mathrm{~s}, 3 \mathrm{H}), 2.78(\mathrm{~s}, 3 \mathrm{H}), 2.69(\mathrm{~s}, 3 \mathrm{H}), 2.68(\mathrm{~s}, 3 \mathrm{H}) ;{ }^{13} \mathrm{C}$ NMR $\left(\mathrm{CDCl}_{3}, 100 \mathrm{MHz}\right): \delta 161.9,161.5,160.9,160.6,143.1,142.4,142.3,142.1,141.8$, 139.3, 131.1, 130.8, 129.9, 127.5, 127.3, 127.1, 127.0, 126.8, 126.6, 126.5, 126.1, 126.0, $125.9,125.8,125.7,125.6,125.5,125.2,125.0,124.4,122.6,112.9,112.5,41.0,40.5$, 
40.2, 40.1, 40.0, 39.6, 39.5, 39.2, 38.4, 31.1. HRMS (EI) calcd for C54H55O4N9: 893.4372; found 893.4347.

Urea 5. $1 \%$ overall-yield. mp $108-111{ }^{\circ} \mathrm{C} ;{ }^{1} \mathrm{H}$ NMR $\left(\mathrm{CDCl}_{3}, 500 \mathrm{MHz}\right): \delta 8.10-7.81$ (m, 7H), $7.72(\mathrm{~d}, J=9 \mathrm{~Hz}, 1 \mathrm{H}), 7.44(\mathrm{~d}, J=7.5 \mathrm{~Hz}, 2 \mathrm{H}), 6.54-6.07(\mathrm{~m}, 18 \mathrm{H}), 5.76(\mathrm{~d}, J=$ $8.0 \mathrm{~Hz}, 2 \mathrm{H}), 3.38(\mathrm{~s}, 3 \mathrm{H}), 3.05-2.89(\mathrm{~m}, 21 \mathrm{H}), 2.78(\mathrm{~s}, 3 \mathrm{H}), 2.67(\mathrm{~s}, 3 \mathrm{H}), 1.87(\mathrm{~s}, 3 \mathrm{H}){ }^{13}$ C NMR $\left(\mathrm{CDCl}_{3}, 100 \mathrm{MHz}\right): \delta 161.9,161.5,160.9,160.8,160.5,143.1,142.5,142.4$, $142.3,142.1,141.8,139.3,135.8,131.1,130.8,129.8,127.5,127.3,127.1,127.0,126.8$, $126.6,126.5,126.0,125.9,125.9,125.8,125.7,125.5,125.2,125.0,124.4,122.6,112.6$, 41.2, 40.5, 40.2, 40.1, 40.0, 39.5, 39.4, 39.2, 38.4, 31.2. MS (EI) for C63H65O5N11: found 1055.2.

Urea 6. $15 \%$ overall-yield. $\mathrm{mp} 232-236{ }^{\circ} \mathrm{C} ;{ }^{1} \mathrm{H}$ NMR $\left(\mathrm{CDCl}_{3}, 500 \mathrm{MHz}\right): \delta 8.10-7.82$ (m, 7H); $7.73(\mathrm{~d}, \mathrm{~J}=9 \mathrm{~Hz}, 1 \mathrm{H}) ; 7.44(\mathrm{~d}, J=8 \mathrm{~Hz}, 1 \mathrm{H}) ; 6.94-6.84(\mathrm{~m}, 3 \mathrm{H}), 6.65(\mathrm{~d}, J=$ $7.5 \mathrm{~Hz}, 2 \mathrm{H}), 6.28(\mathrm{~d}, J=7.5 \mathrm{~Hz}, 2 \mathrm{H}), 6.13(\mathrm{~d}, J=8.0 \mathrm{~Hz}, 2 \mathrm{H}), 6.08(\mathrm{~d}, J=8.0 \mathrm{~Hz}, 2 \mathrm{H})$, $5.78(\mathrm{~d}, J=7.5 \mathrm{~Hz}, 2 \mathrm{H}), 3.39$ (s, 3H), 3.08 (s, 3H), 3.04 (s, 3H), $2.96(\mathrm{~s}, 3 \mathrm{H}), 2.74(\mathrm{~s}$,

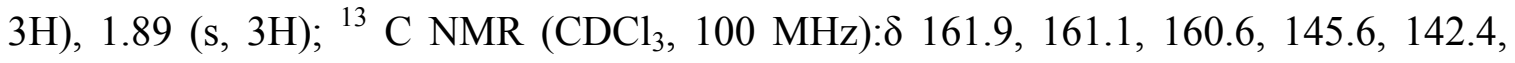
$142.3,142.2,141.8,139.4,131.1,130.8,129.8,128.8,127.5,126.8,126.6,126.0,125.9$, $125.8,125.2,125.1,124.4,40.5,40.2,39.6,39.5,39.2,39.4$. HRMS (EI) calcd for C43H40O3N6: 688.3150; found 688.3156.

Femtosecond broadband pump-probe spectroscopy. A detailed description of our experimental setup has been given elsewhere. ${ }^{4}$ The pump wavelength was set to $355 \mathrm{~nm}$ for all ureas. The changes in optical density were probed by a femtosecond white-light continuum (WLC) generated by tight focusing of a small fraction of the output of a commercial Ti:Sp based pump laser (CPA-2010, Clark-MXR) into a $3 \mathrm{~mm}$ calcium 
fluoride $\left(\mathrm{CaF}_{2}\right)$ plate. The WLC provides a usable probe source between 300 and $750 \mathrm{~nm}$. The WLC was split into two beams (probe and reference) and focused into the sample using reflective optics. After passing through the sample both probe and reference beams were spectrally dispersed and simultaneously detected on a CCD sensor. The pump pulse $(1 \mathrm{kHz}, 400 \mathrm{~nJ})$ was generated by frequency doubling of the compressed output of a home-built NOPA system (from $666 \mathrm{~nm}$ to $708 \mathrm{~nm}$ respectively, $7 \mu \mathrm{J}, 40 \mathrm{fs}$ ). To compensate for group velocity dispersion in the UV-pulse an additional prism compressor was used. The overall time resolution of the setup is determined by the cross correlation function between pump and probe pulses which is typically 100-120 fs (fwhm, assuming a Gaussian lineshape). A spectral resolution of 5-7 nm was obtained. All measurements were performed with magic angle $\left(54.7^{\circ}\right)$ setting for the polarization of pump with respect to the polarization of the probe pulse. A sample cell with $1.25 \mathrm{~mm}$ fused silica windows and an optical path of $1 \mathrm{~mm}$ was used for all measurements. A wire stirrer was used to ensure fresh sample volume was continuously used during the measurement. 


\section{Absorption Spectra}

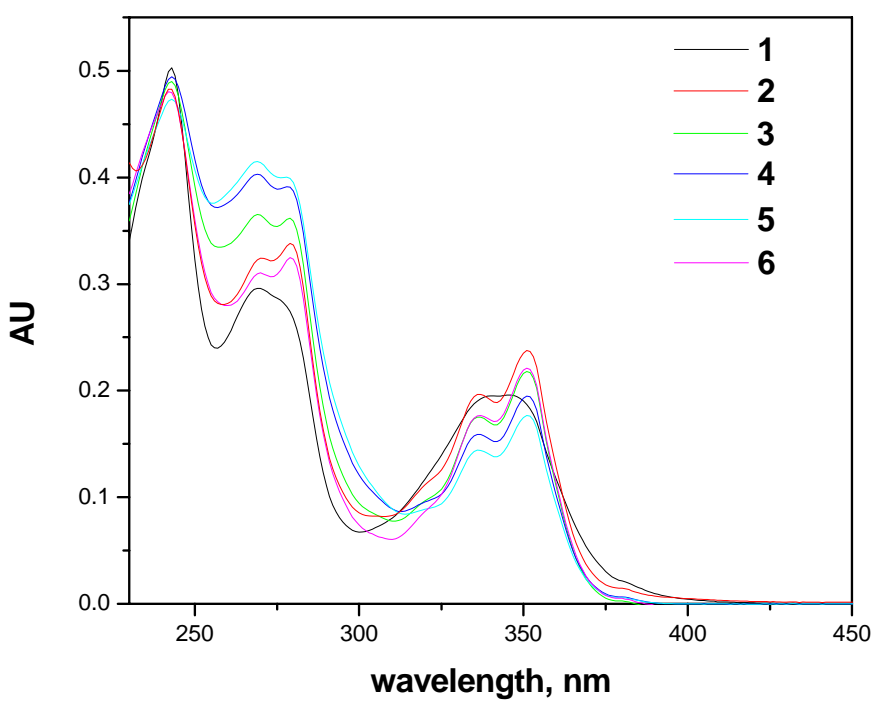

Figure A. Absorption spectra of ureas 1-6 in acetonitrile. 


\section{Transient Spectra}

(a)

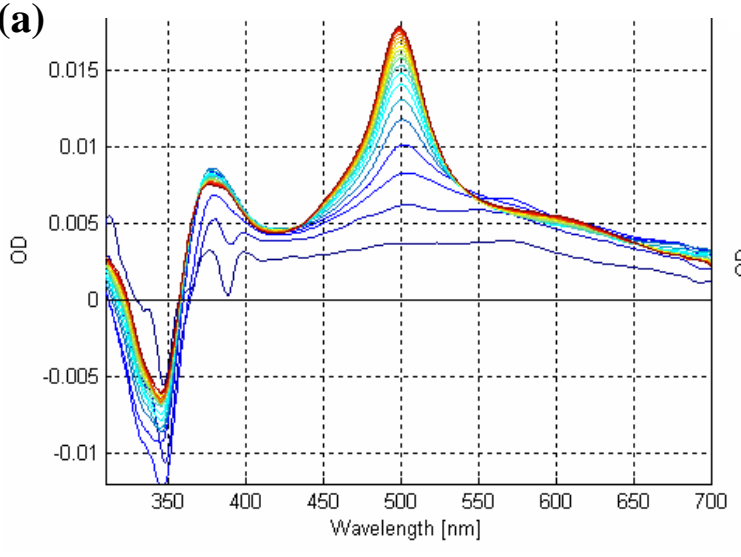

(b)

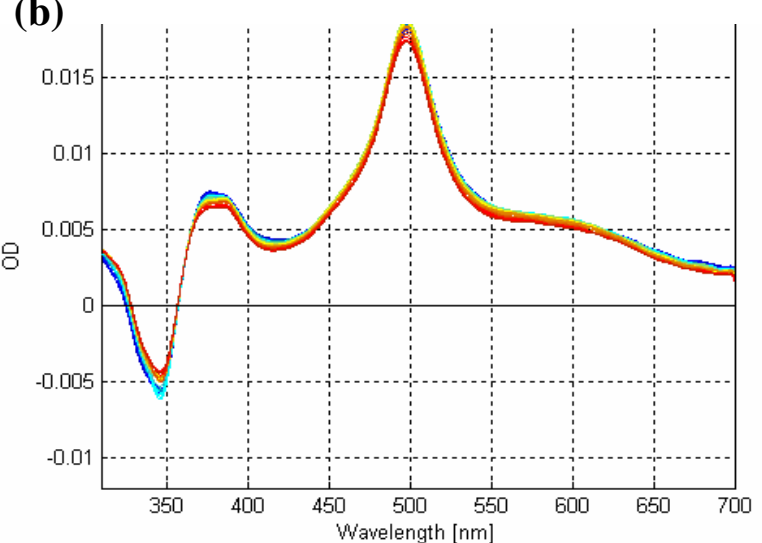

(c)

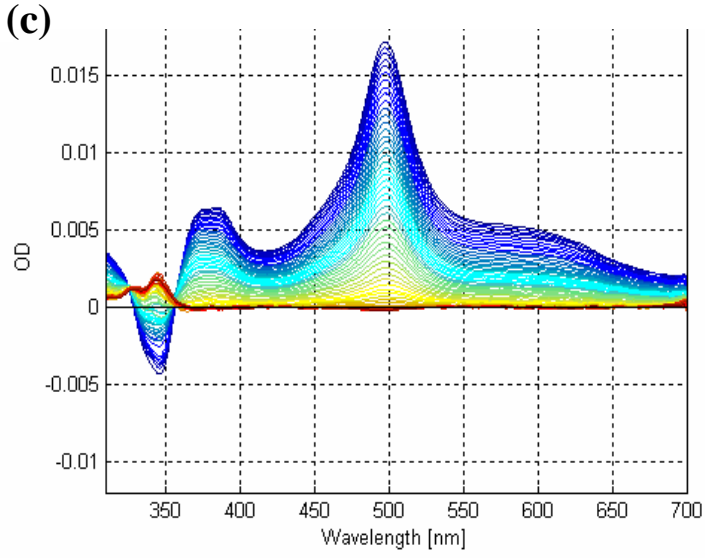

Figure B. Temporal evolution of the pump-probe spectra of urea 1 (a) 0-1 ps, (b) 1-5 ps, (c) 5-2000 ps in the indicated time range following $347 \mathrm{~nm}$ excitation in Acetonitrile. Early spectra are shown in blue/green colors and late spectra in orange/red colors.

(a)

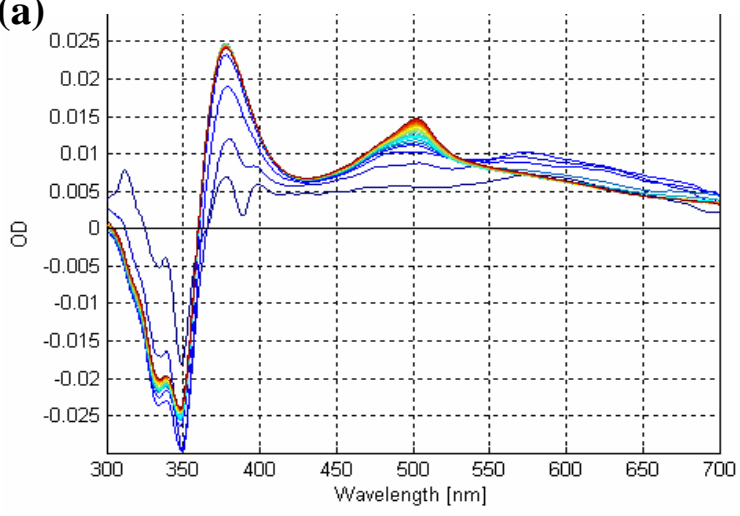

(b)

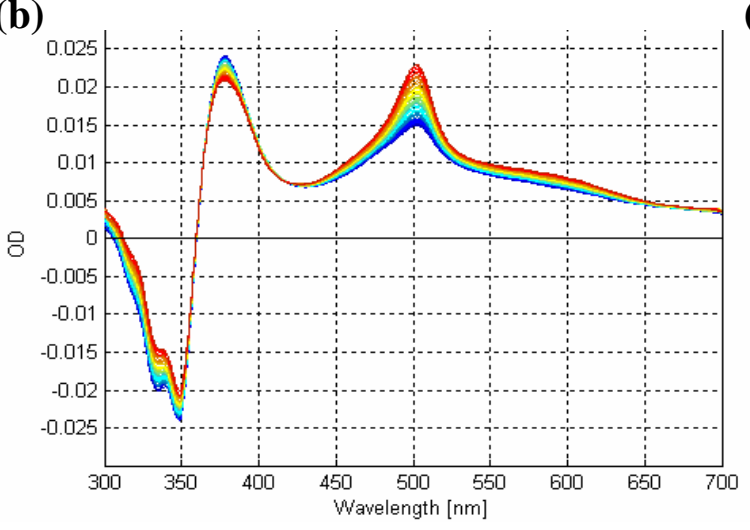

(c)

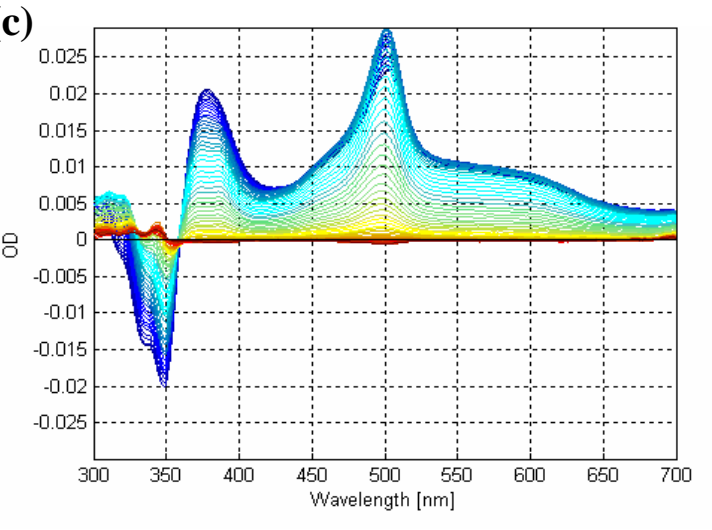

Figure C. Temporal evolution of the pump-probe spectra of urea 2 (a) 0-1 ps, (b) 1-5 ps, (c) 5-2000 ps in the indicated time range following $347 \mathrm{~nm}$ excitation in Acetonitrile. Early spectra are shown in blue/green colors and late spectra in orange/red colors. 
(a)

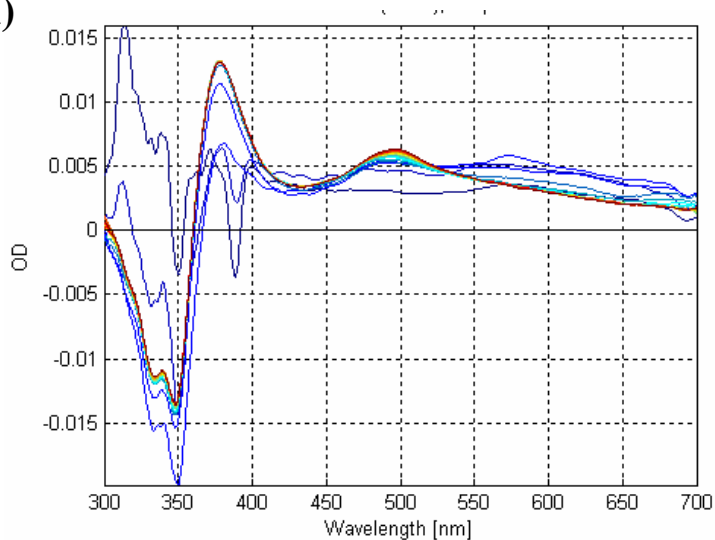

(b)

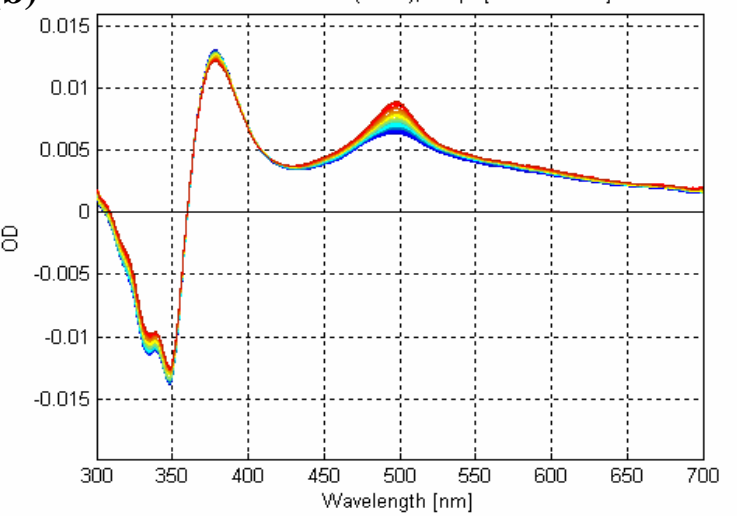

(c)

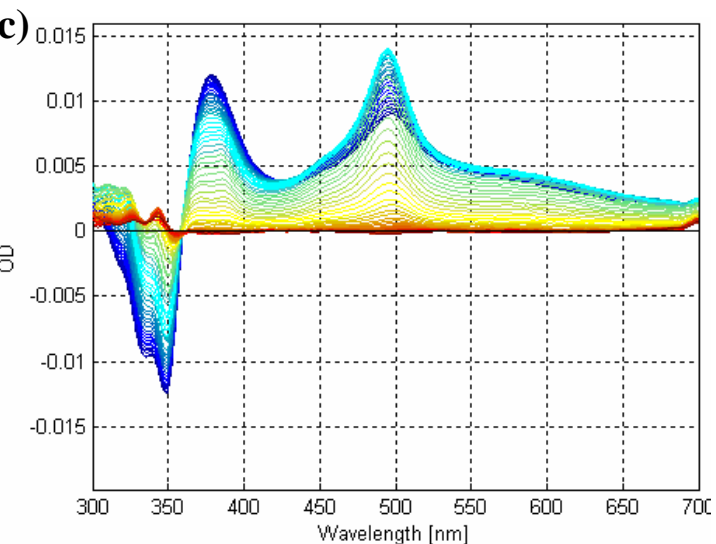

Figure D. Temporal evolution of the pump-probe spectra of urea 3 (a) 0-1 ps, (b) 1-5 ps, (c) 5-2000 ps in the indicated time range following $347 \mathrm{~nm}$ excitation in Acetonitrile. Early spectra are shown in blue/green colors and late spectra in orange/red colors.

(a)

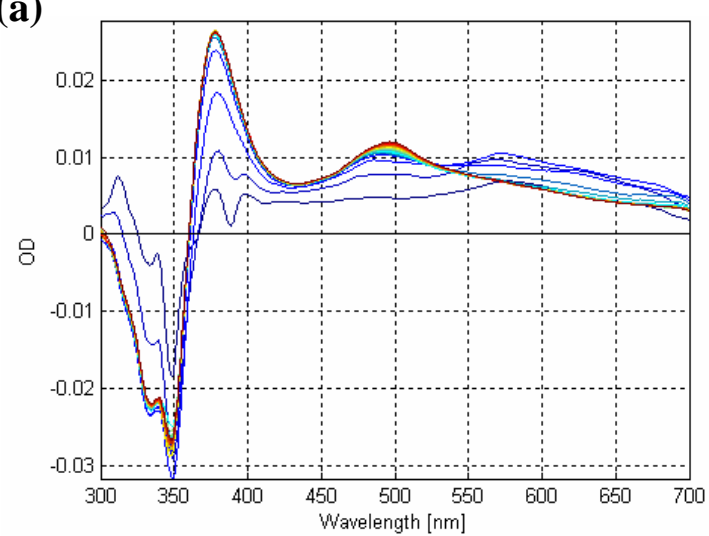

(b)

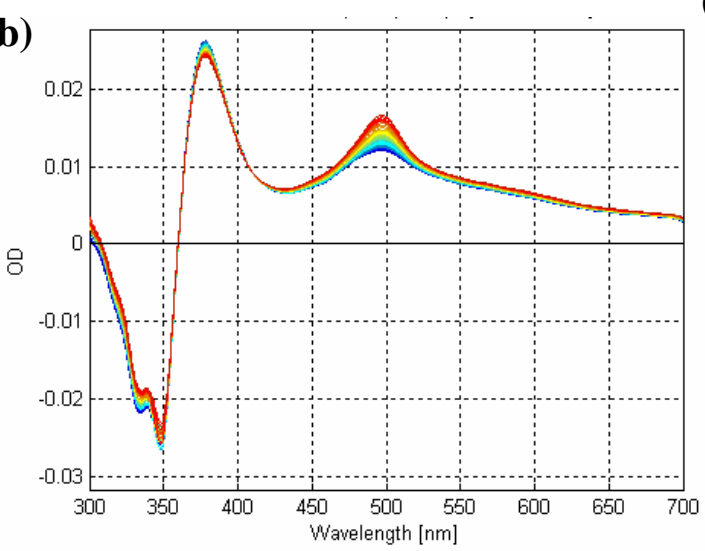

(c)

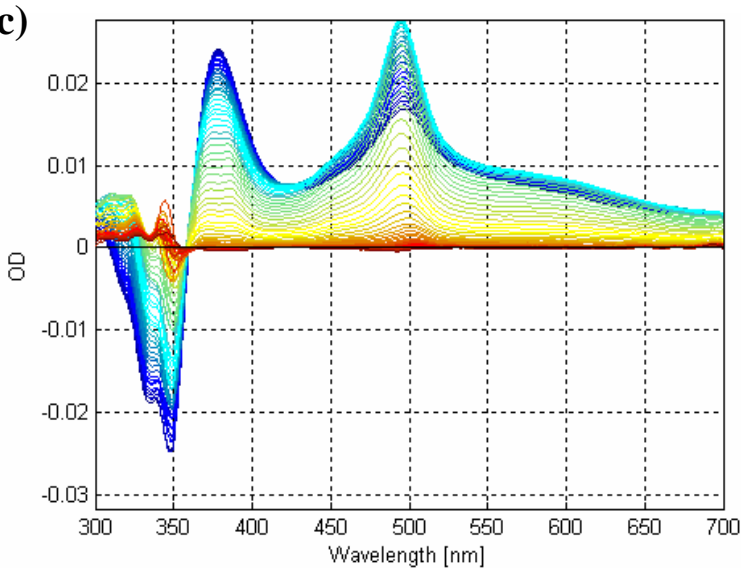

Figure E. Temporal evolution of the pump-probe spectra of urea 4 (a) 0-1 ps, (b) 1-5 ps, (c) 5-2000 ps in the indicated time range following $347 \mathrm{~nm}$ excitation in Acetonitrile. Early spectra are shown in blue/green colors and late spectra in orange/red colors. 

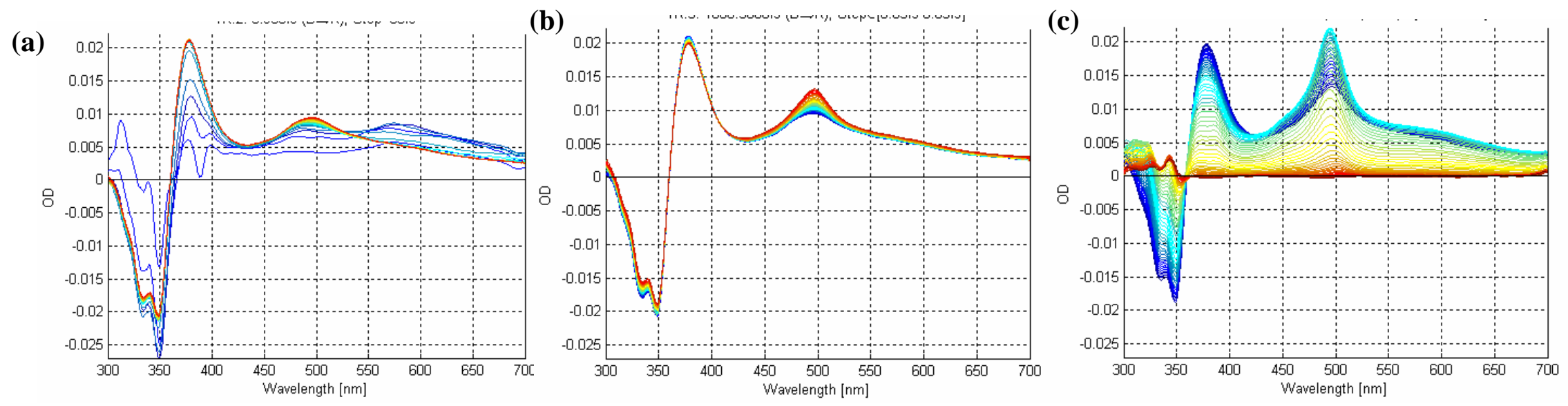

Figure F. Temporal evolution of the pump-probe spectra of urea 5 (a) 0-1 ps, (b) 1-5 ps, (c) 5-2000 ps in the indicated time range following $347 \mathrm{~nm}$ excitation in Acetonitrile. Early spectra are shown in blue/green colors and late spectra in orange/red colors.
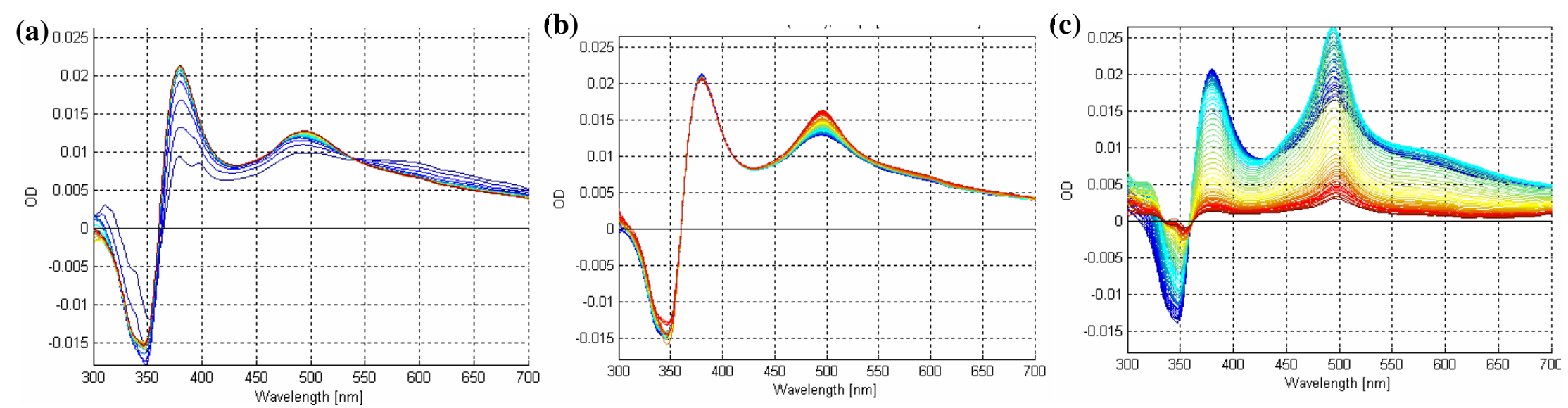

Figure J. Temporal evolution of the pump-probe spectra of urea 6 (a) 0-1 ps, (b) 1-5 ps, (c) 5-2000 ps in the indicated time range following $347 \mathrm{~nm}$ excitation in Acetonitrile. Early spectra are shown in blue/green colors and late spectra in orange/red colors. 


\section{ZINDO Calculations}
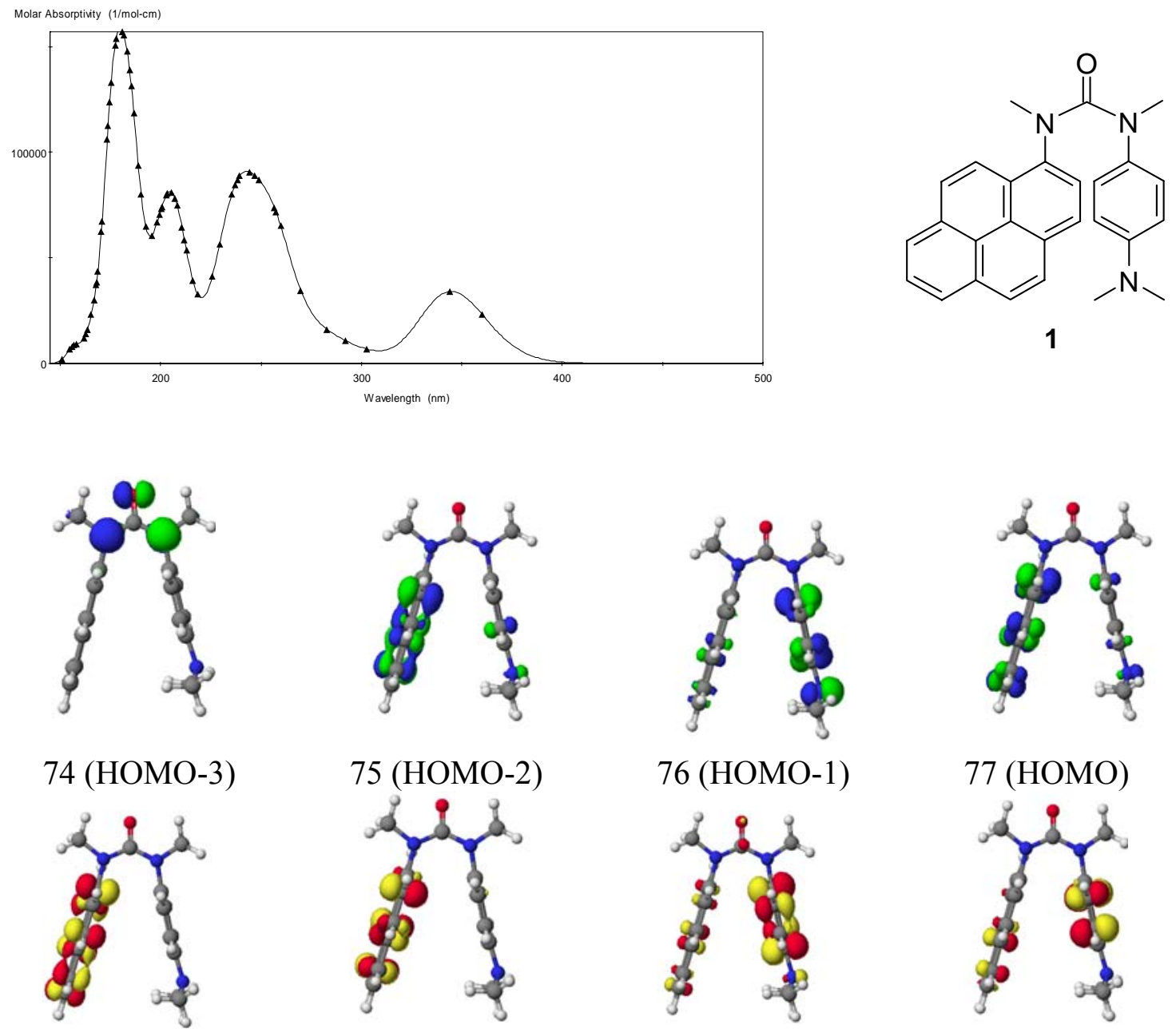

77 (HOMO)

78 (LUMO)

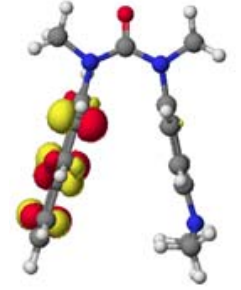

$79(\mathrm{LUMO}+1)$

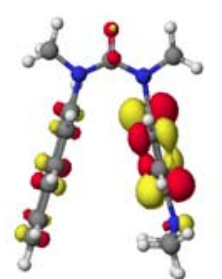

$80(\mathrm{LUMO}+2)$

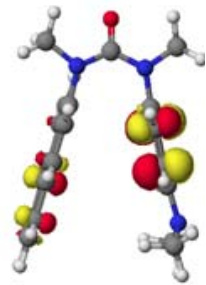

$81(\mathrm{LUMO}+3)$

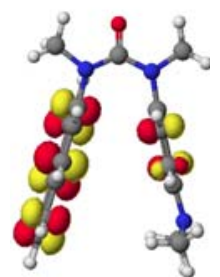

$82(\mathrm{LUMO}+4)$

AM1 minimizes geometries Transitions $\mathrm{w} / \mid$ coeff $\mid>0.250, \mathrm{f}\left(\mathrm{M}^{\wedge}-1 * \mathrm{~cm}^{\wedge}-1\right)$ $\mathrm{S} 1: 360.0 \mathrm{~nm}$, $\mathrm{S} 2: 344.0 \mathrm{~nm}$, $\mathrm{S} 3: 302.4 \mathrm{~nm}$, $\mathrm{S} 4: 292.2 \mathrm{~nm}$,

75->78 $-0.553$ $77->78$

$-0.914$

$76->81$

0.466

76- $>81$

0.314

$77->78$

$-0.272$

$77->79$

$76->83$

$-0.307$

$77->80$

$-0.261$

$77->79 \quad 0.714$

$77->81$

$-0.583$

$77->81$

0.256

$77->82$

0.816 


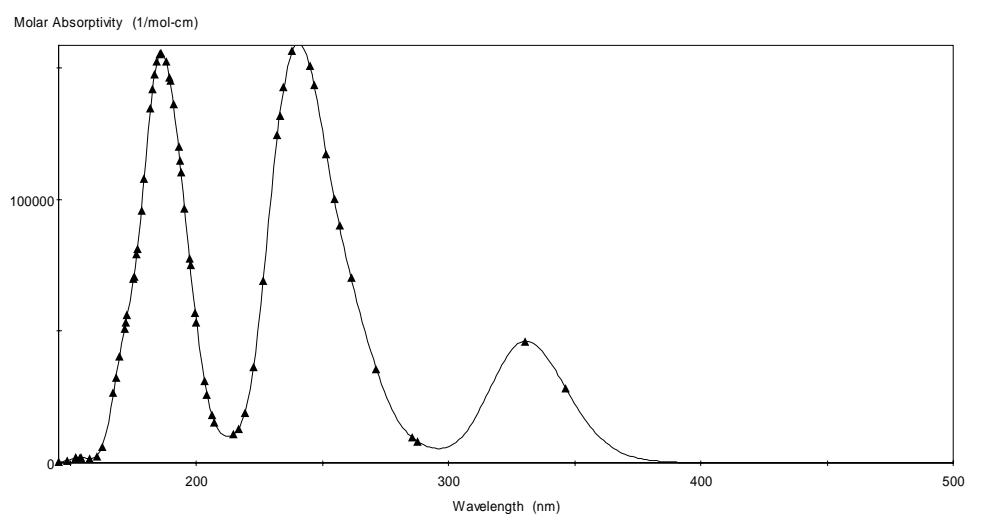<smiles></smiles>
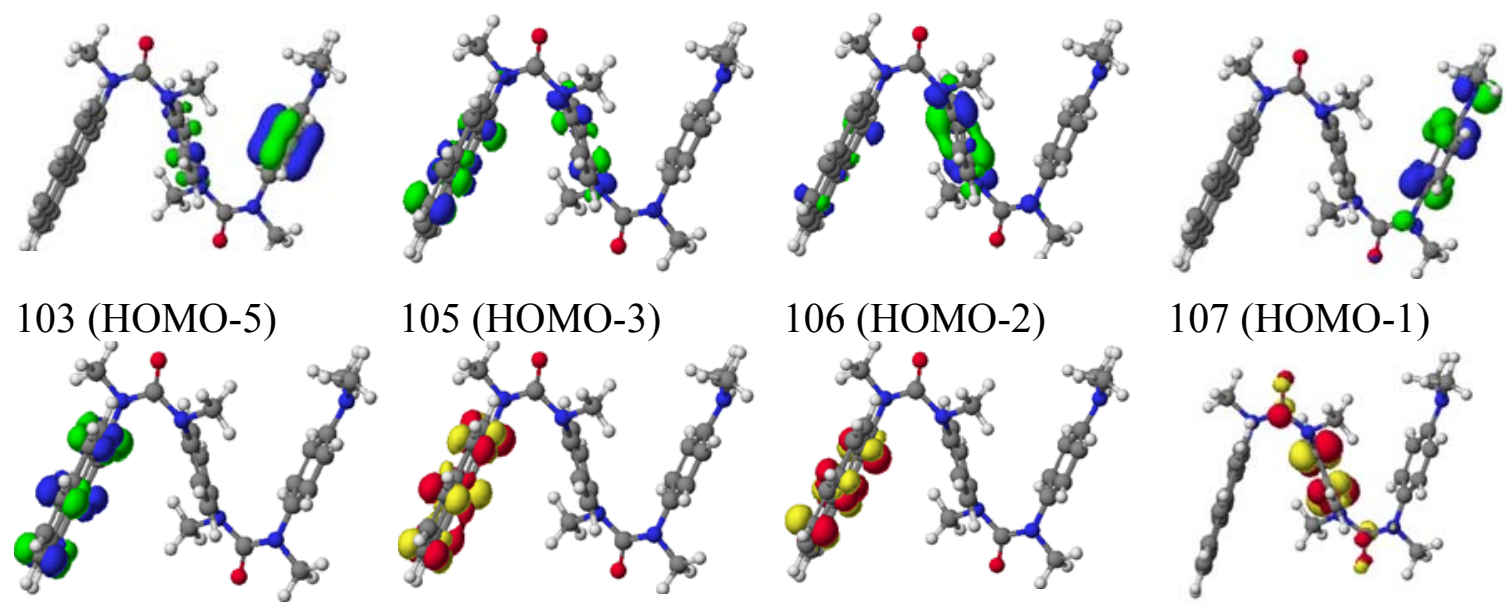

106 (HOMO-2)

107 (HOMO-1)

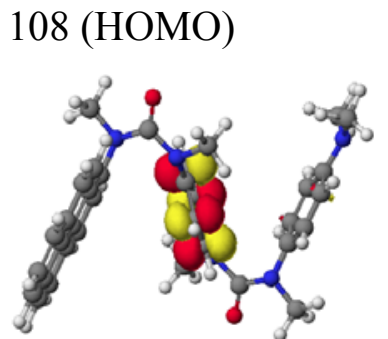

$112(\mathrm{LUMO}+3)$

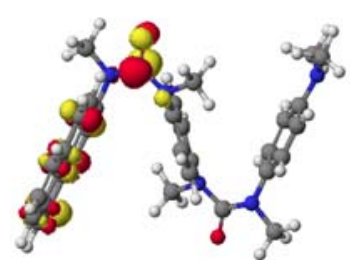

$116(\mathrm{LUMO}+7)$

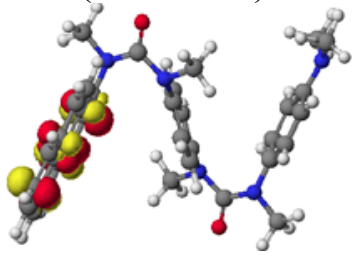

$110(\mathrm{LUMO}+1)$

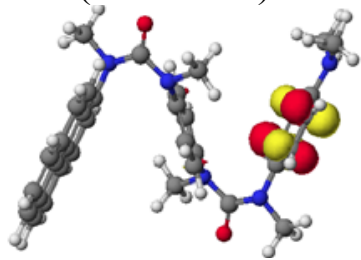

$114(\mathrm{LUMO}+5)$

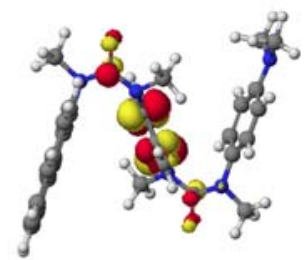

$111(\mathrm{LUMO}+2)$

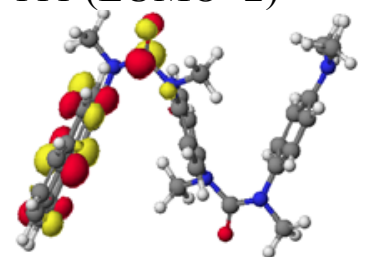

$115(\mathrm{LUMO}+6)$

AM1 minimizes geometries Transitions $\mathrm{w} / \mid$ coeff $\mid>0.250, \mathrm{f}\left(\mathrm{M}^{\wedge}-1 * \mathrm{~cm}^{\wedge}-1\right)$

$\mathrm{S} 1: 346.4 \mathrm{~nm}$,

$105->109 \quad 0.483$

$106->109 \quad-0.387$

$108->110 \quad 0.759$
S2: $330.4 \mathrm{~nm}$,

108->109 -0.936

$\mathrm{S} 3: 287.7 \mathrm{~nm}$,

$103->113 \quad 0.341$

$107->112 \quad 0.280$

$107->114 \quad 0.826$
$\mathrm{S} 4: 285.5 \mathrm{~nm}$,

$108->115 \quad 0.850$

$108->116 \quad-0.484$ 

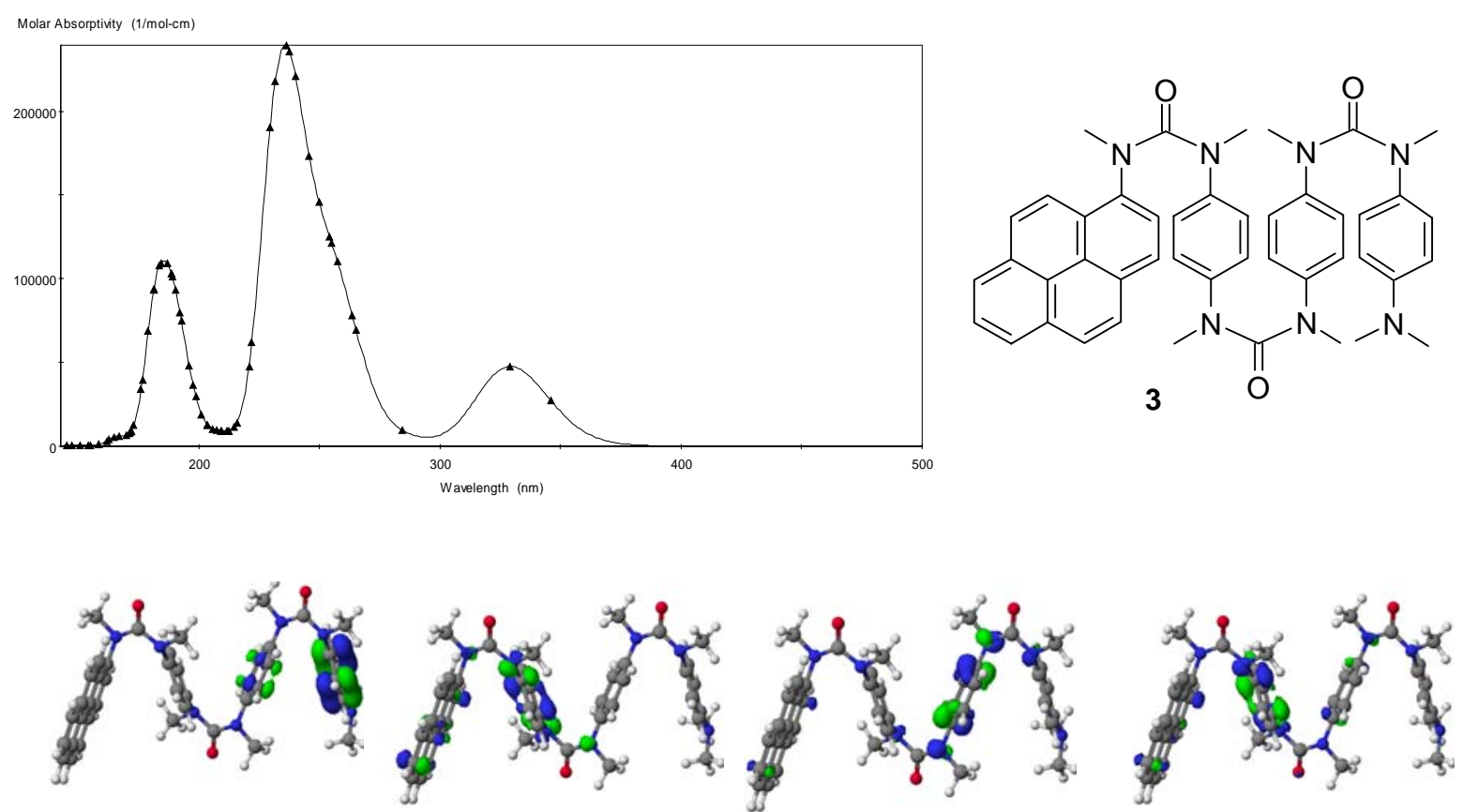

133 (HOMO-6) 135 (HOMO-4)

136 (HOMO-3)

137 (HOMO-2)
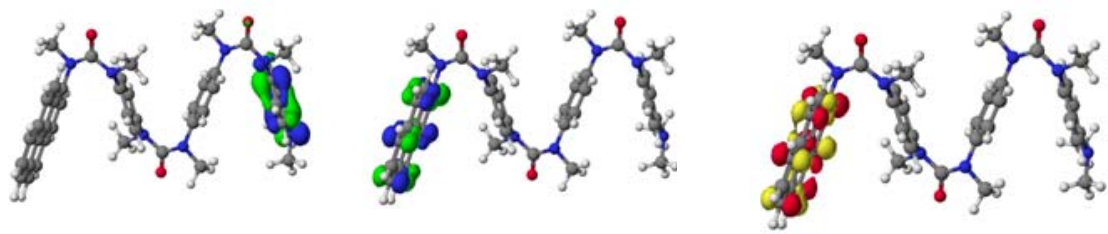

138 (HOMO-1) 139 (HOMO)
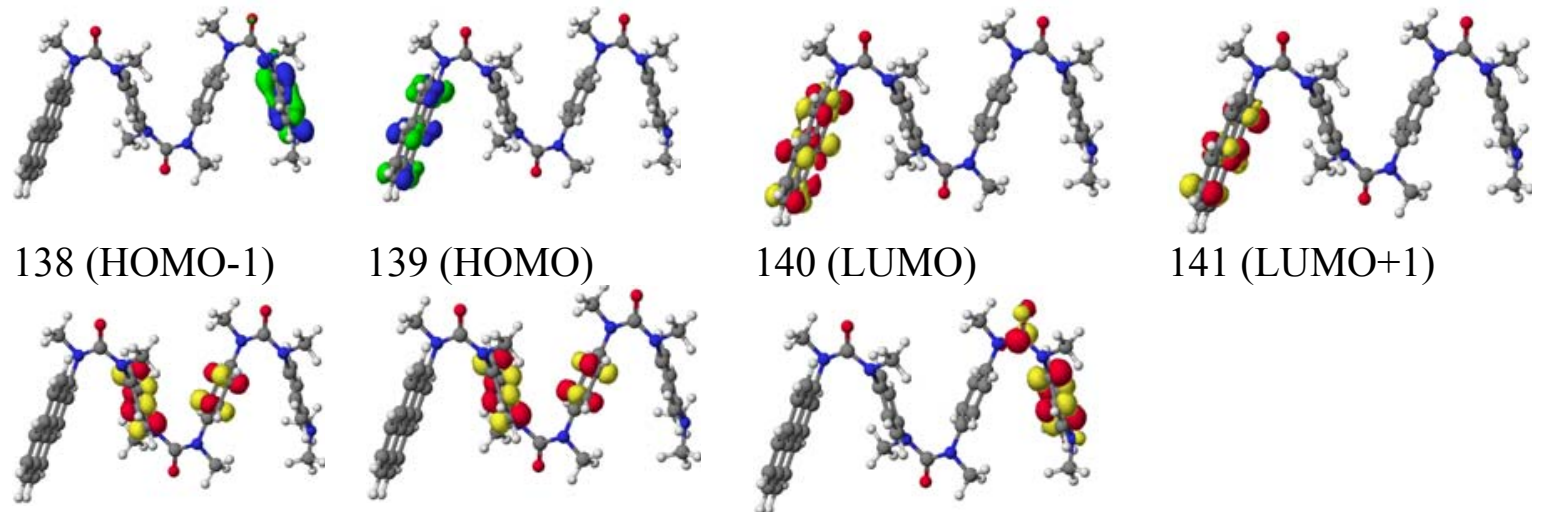

144 (LUMO+4)

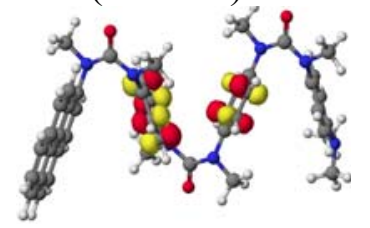

$145(\mathrm{LUMO}+5)$

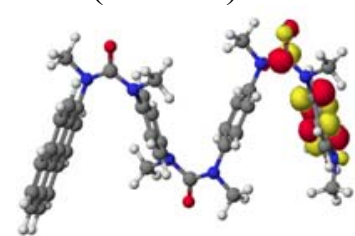

$146(\mathrm{LUMO}+6)$

AM1 minimizes geometries Transitions $\mathrm{w} / \mid$ coeff $\mid>0.250, \mathrm{f}\left(\mathrm{M}^{\wedge}-1 * \mathrm{~cm}^{\wedge}-1\right)$

$\mathrm{S} 1: 345.9 \mathrm{~nm}$,

$135->140 \quad-0.375$

$136->140 \quad-0.320$

$137->140 \quad-0.378$

$139->141 \quad 0.760$
$\mathrm{S} 2: 329.1 \mathrm{~nm}$,

139->140 0.939
$\mathrm{S} 3: 284.3 \mathrm{~nm}$,

$133->146 \quad-0.330$

$138->146 \quad 0.262$
S4: $265.2 \mathrm{~nm}$,

$135->145 \quad 0.353$

$136->144 \quad 0.283$

$136->145 \quad-0.351$

$137->144 \quad-0.522$ 

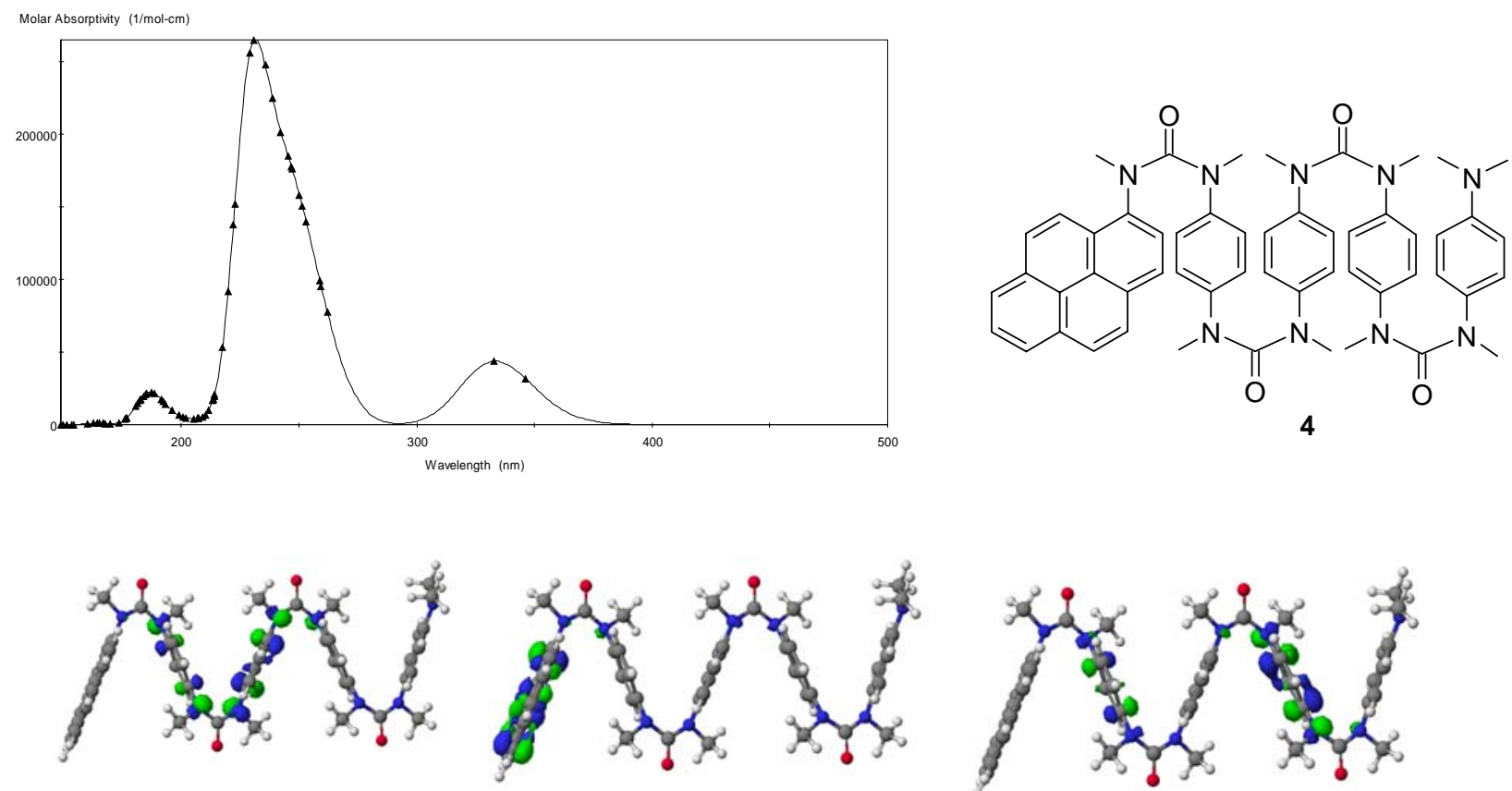

165 (HOMO-5)

166 (HOMO-4)
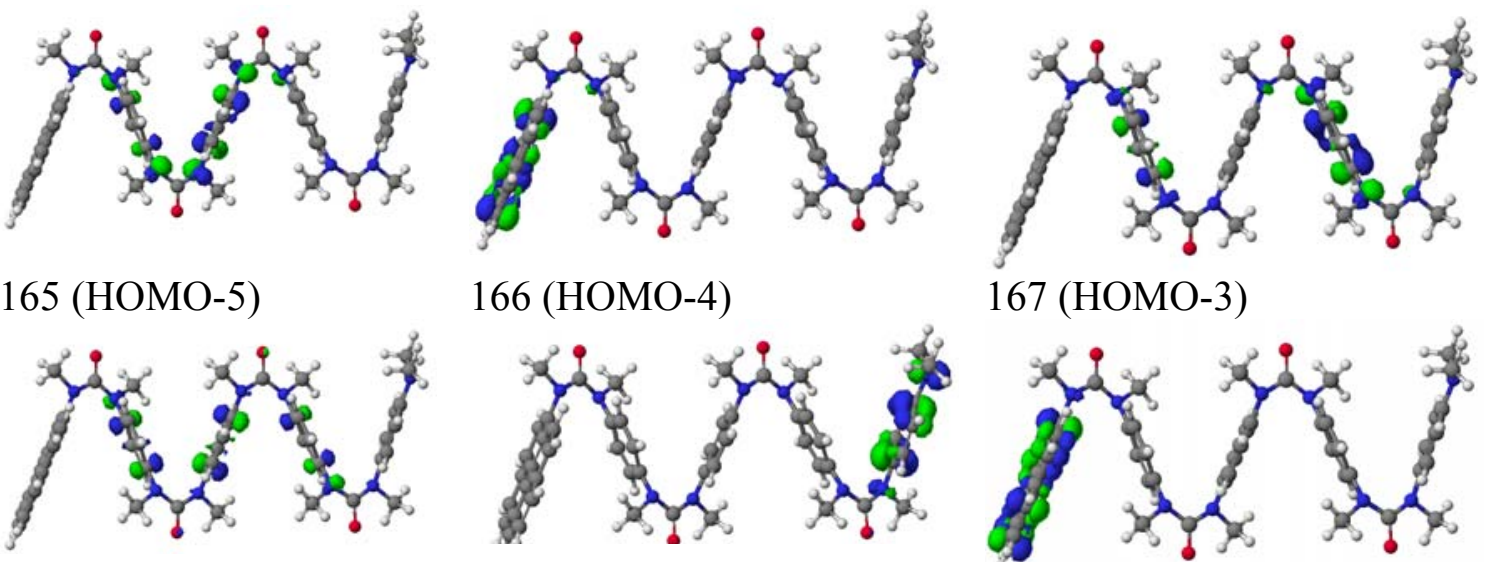

168 (HOMO-2)

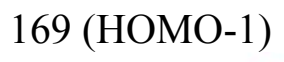

167 (HOMO-3)
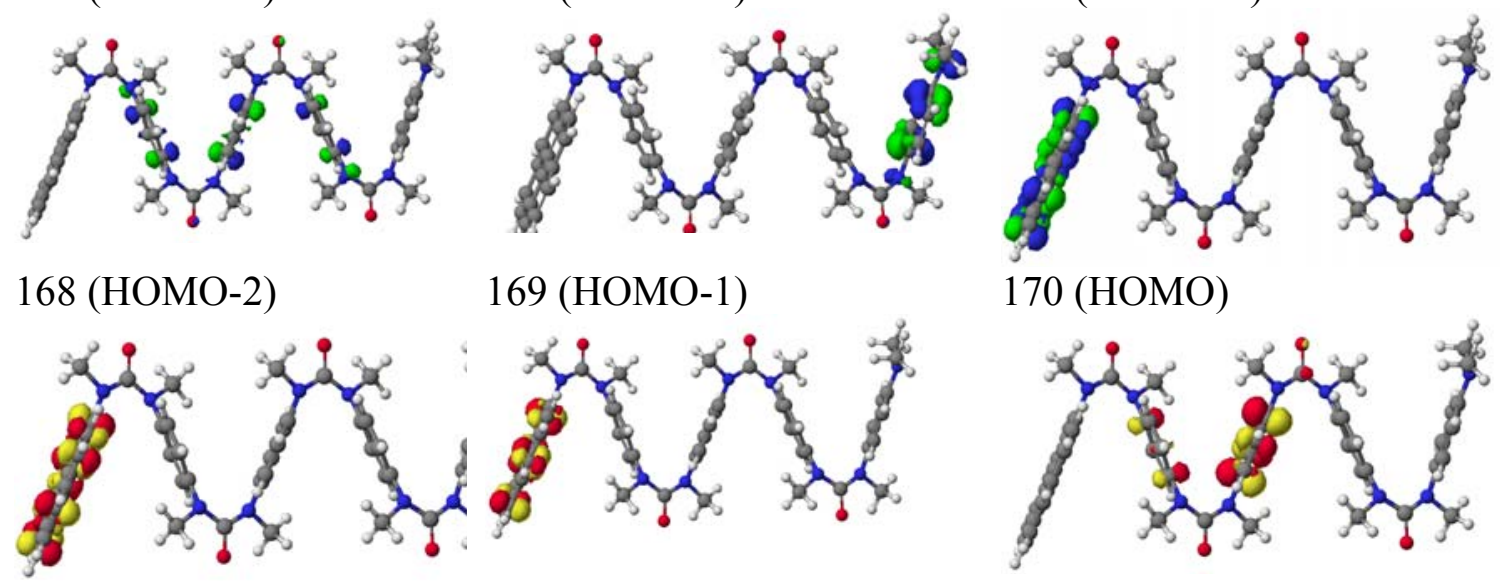

171 (LUMO)

172 (LUMO+1)

170 (HOMO)

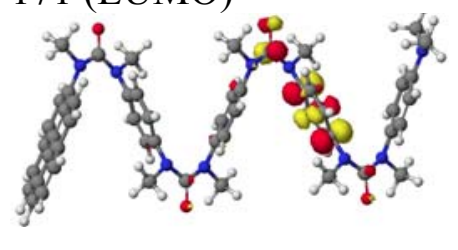

175 (LUMO+4)

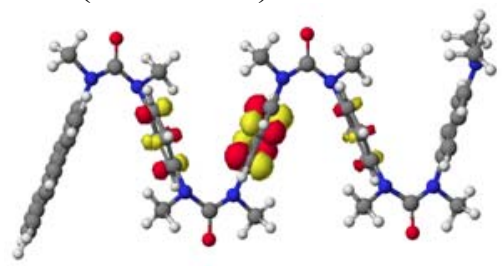

$176(\mathrm{LUMO}+5)$

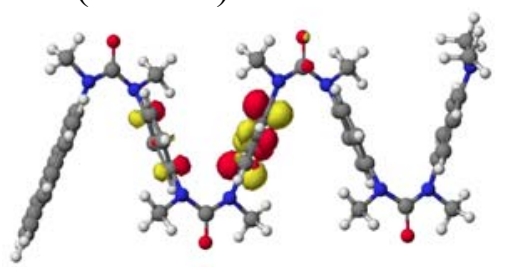

173 (LUMO+2)

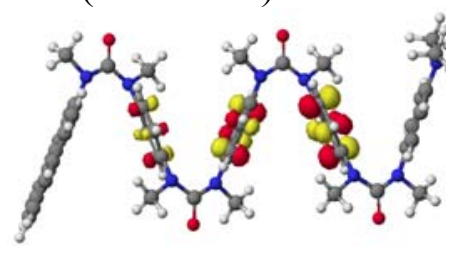

178 (LUMO+7)

AM1 minimizes geometries $\quad$ Transitions $\mathrm{w} / \mid$ coeff $\mid>0.250, \mathrm{f}\left(\mathrm{M}^{\wedge}-1 * \mathrm{~cm}^{\wedge}-1\right)$

$\mathrm{S} 1: 346.2 \mathrm{~nm}$,

$166->171 \quad 0.550$

$170->171 \quad-0.211$

$170->172 \quad-0.751$
S2: $332.9 \mathrm{~nm}$,

$166->172 \quad 0.245$

$170->171 \quad-0.930$
$\mathrm{S} 3: 262.0 \mathrm{~nm}$,

$166->171 \quad 0.289$

$168->173 \quad 0.283$

$168->176 \quad-0.274$

$170->172-0.307$
$\mathrm{S} 4: 259.4 \mathrm{~nm}$,

$165->178 \quad-0.322$

$167->177 \quad 0.262$

$168->173 \quad-0.347$

$168->176 \quad-0.471$ 

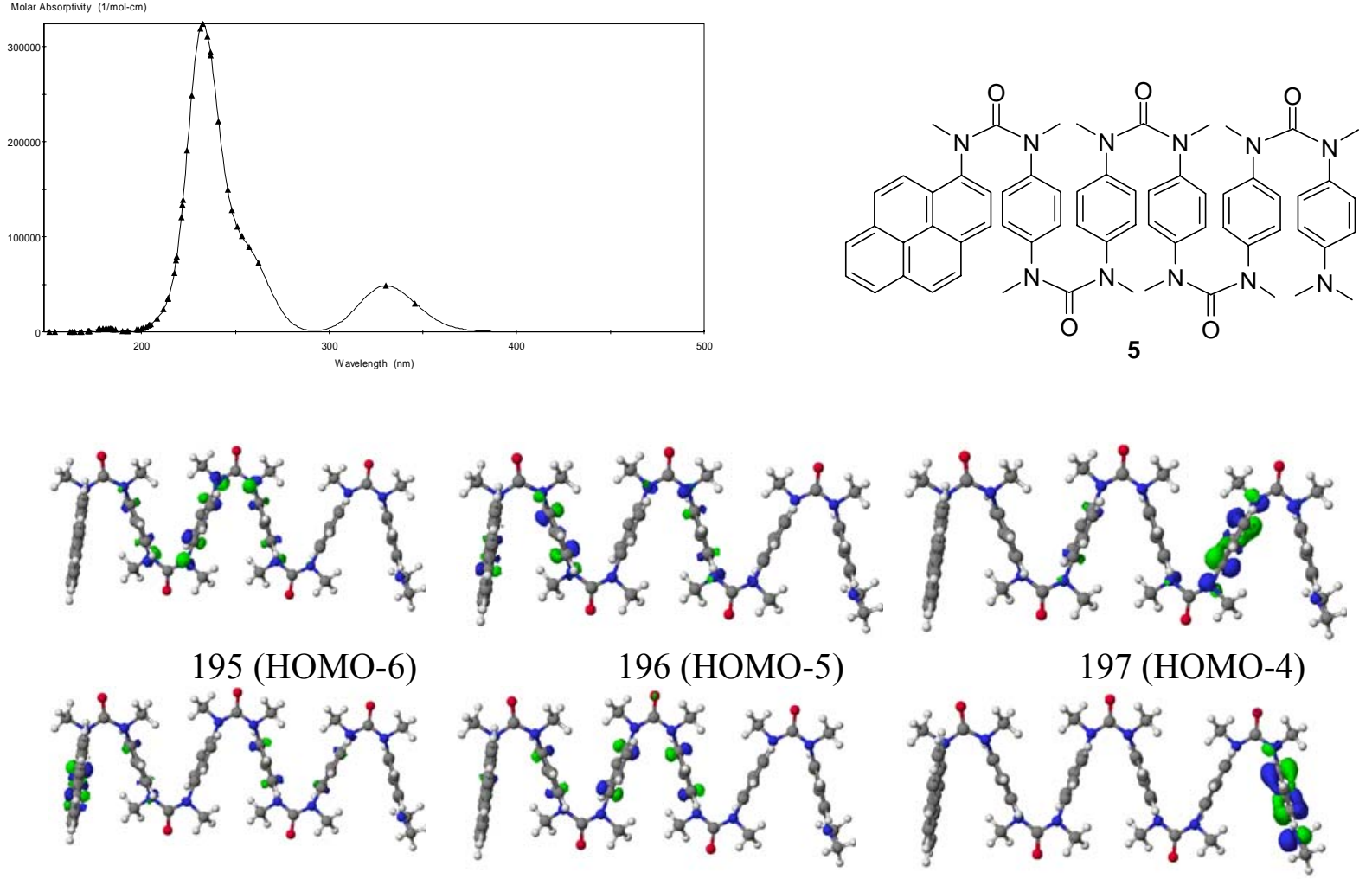

197 (HOMO-4)

$198(\mathrm{HOMO}-3)$

199 (HOMO-2)
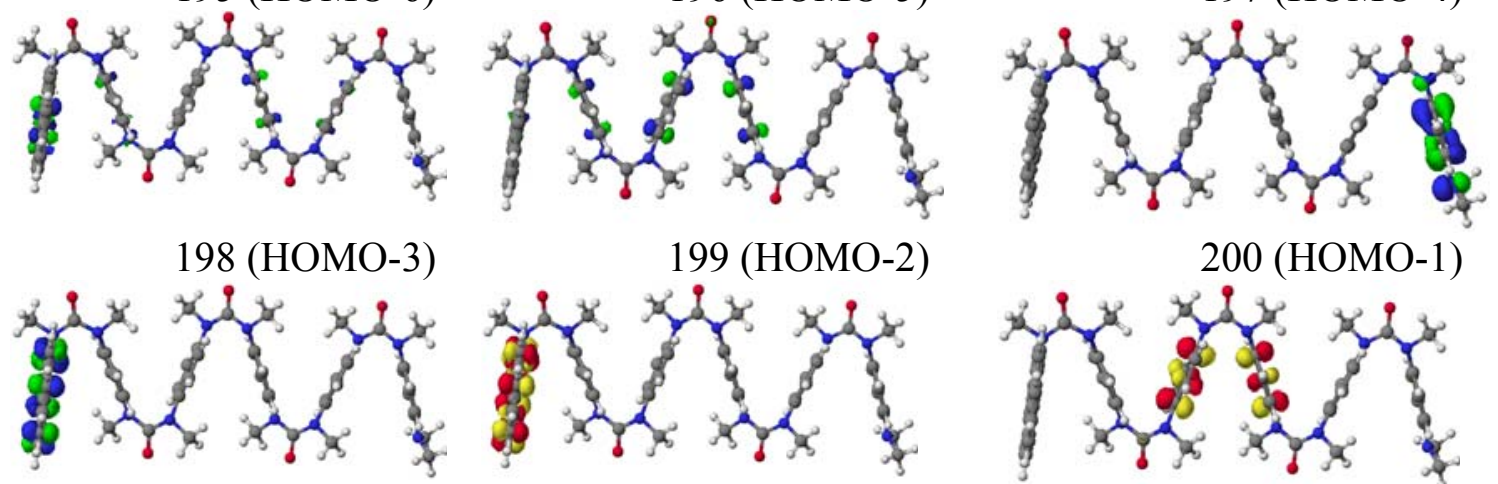

200 (HOMO-1)
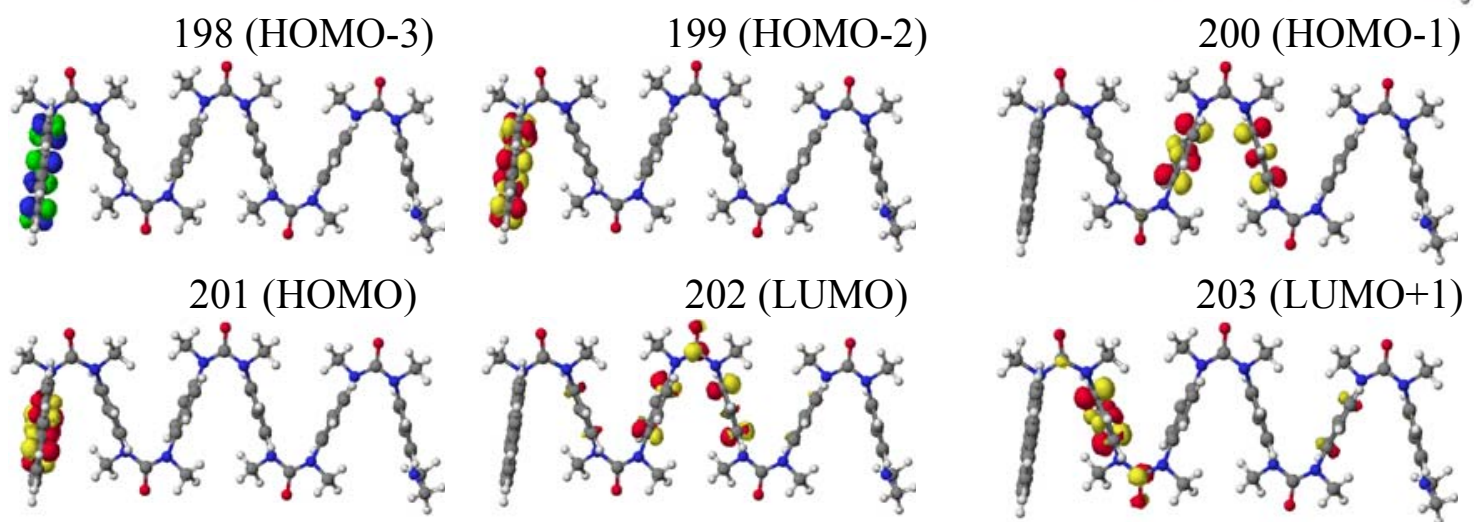

$205(\mathrm{LUMO}+3)$
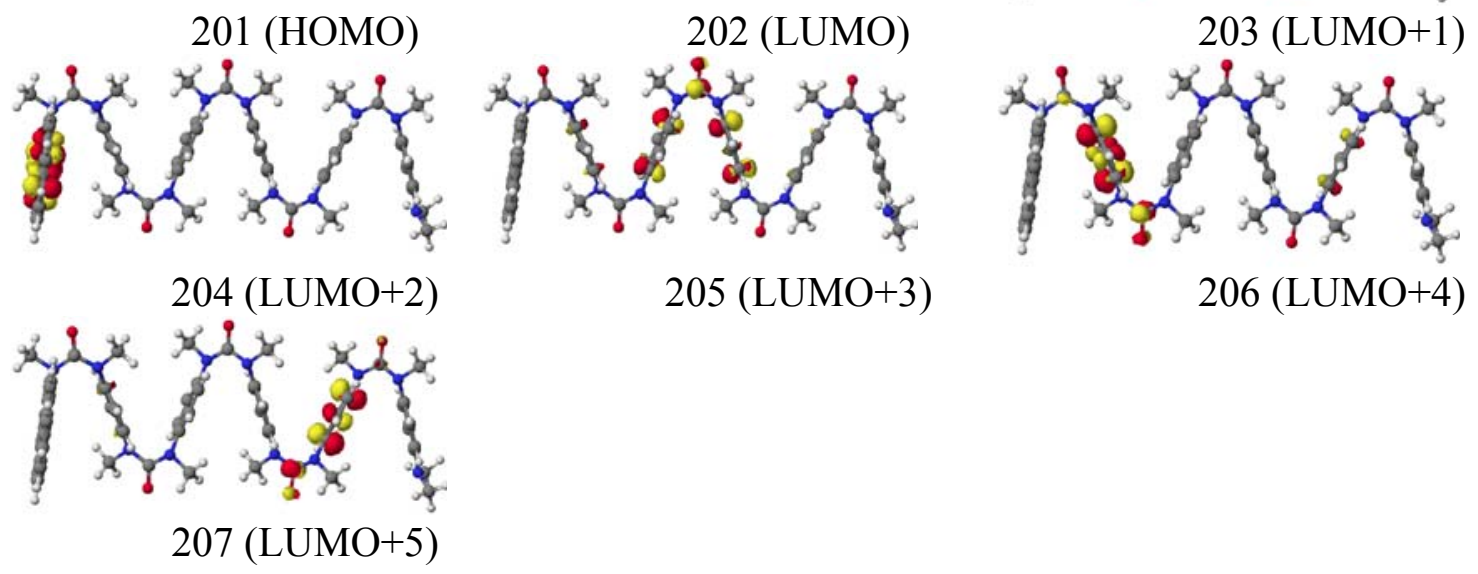

AM1 minimizes geometries $\quad$ Transitions $\mathrm{w} / \mid$ coeff $\mid>0.250, \mathrm{f}\left(\mathrm{M}^{\wedge}-1 * \mathrm{~cm}^{\wedge}-1\right)$

$\mathrm{S} 1: 345.9 \mathrm{~nm}$,

196->202 0.308

$198->202 \quad 0.409$

$199->202 \quad 0.269$

201->204 0.764
$\mathrm{S} 2: 330.0 \mathrm{~nm}$,

201->202 0.937
$\mathrm{S} 3: 262.1 \mathrm{~nm}$,

$195->206 \quad 0.270$

$196->205 \quad-0.264$

$199->203 \quad 0.412$

201->206 -0.307
S4: $257.4 \mathrm{~nm}$,

197->207 0.304

$198->202 \quad-0.329$

201->206 0.254 


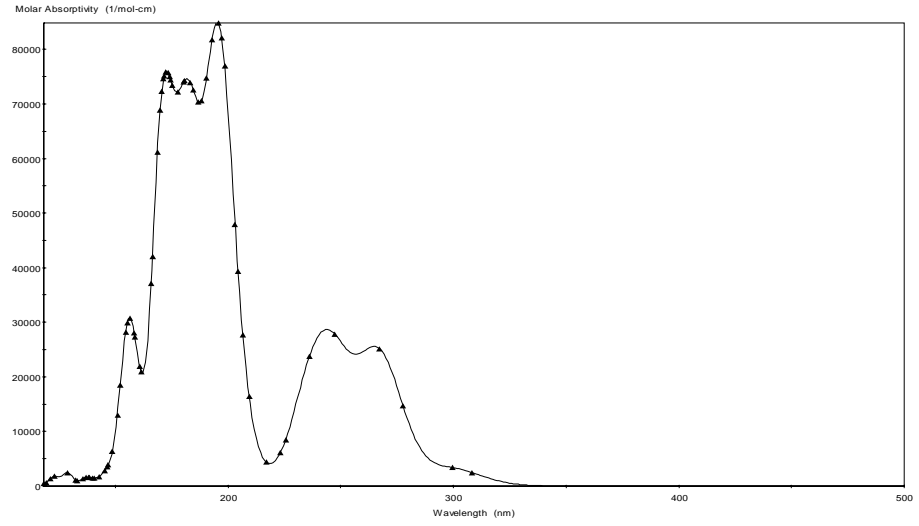<smiles>CN(C)c1ccc(N(C)C(=O)N(C)c2ccccc2)cc1</smiles>

7

\begin{tabular}{|l|l|l|l|}
\hline & & \\
\hline
\end{tabular}

AM1 minimizes geometries

$\mathrm{S} 1: 360.0 \mathrm{~nm}$, $49->57 \quad-0.238$

$49->60 \quad 0.387$

$50->56 \quad 0.264$

$50->57 \quad-0.264$

$50->60 \quad-0.264$

$51->56 \quad-0.264$

$51->57 \quad-0.264$

$51->60 \quad-0.264$

Transitions w/ $\mid$ coeff $\mid>0.250, \mathrm{f}\left(\mathrm{M}^{\wedge}-1 * \mathrm{~cm}^{\wedge}-1\right)$

$\mathrm{S} 1: 360.0 \mathrm{~nm}$,

77->78 $\quad-0.914$

$77->79 \quad 0.714$
$\mathrm{S} 1: 360.0 \mathrm{~nm}$,
$\mathrm{S} 1: 360.0 \mathrm{~nm}, \quad \mathrm{~S} 1: 360.0 \mathrm{~nm}$,

$76->81 \quad 0.466 \quad 76->81 \quad 0.314$

$76->83 \quad-0.307 \quad 77->80 \quad-0.261$

$77->81 \quad-0.583 \quad 77->81 \quad 0.256$ 


\section{NMR Spectra}

H1_std p-500 bb_5m RT Nonsptn

exp1 s2pur
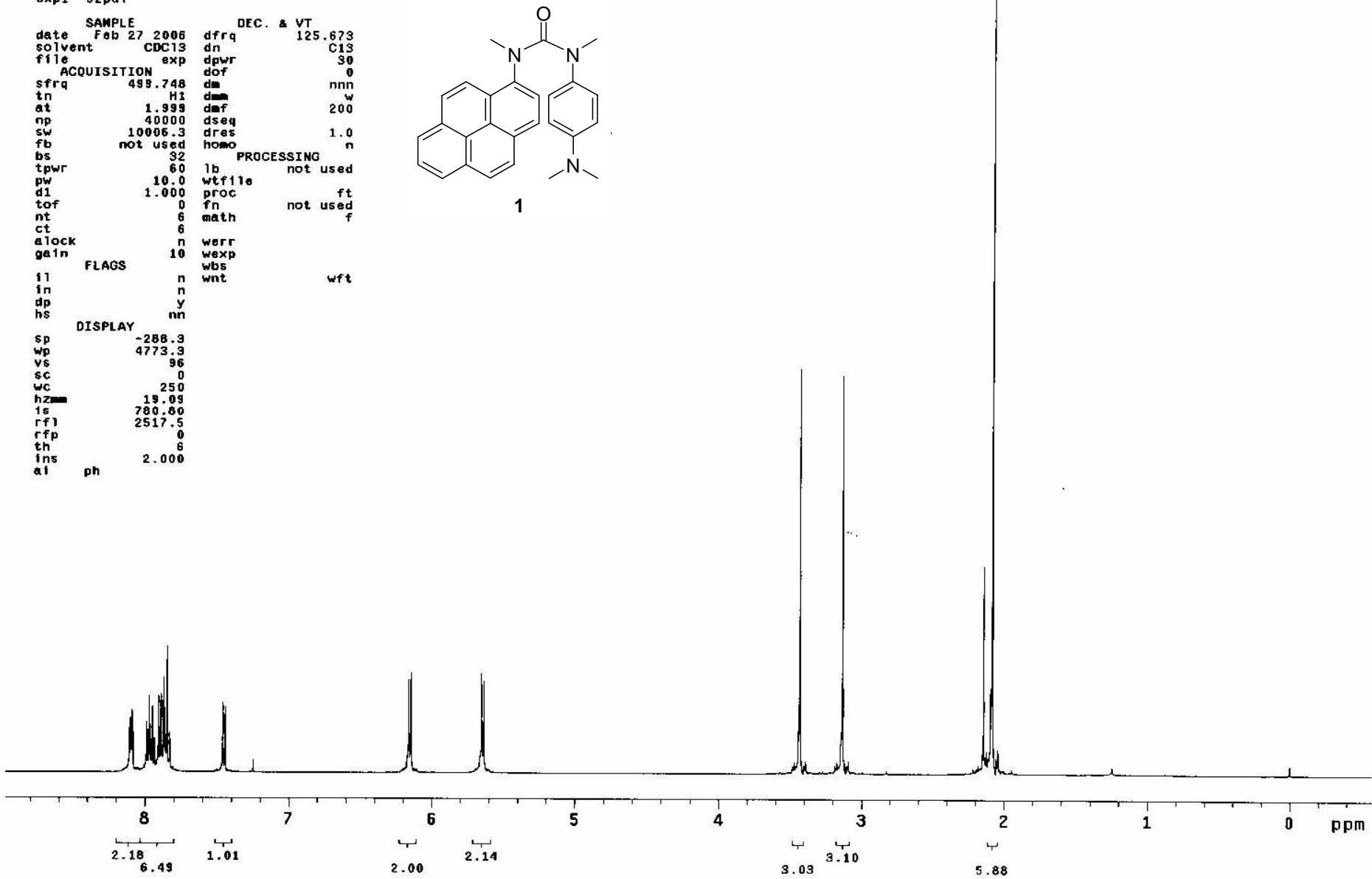
inova 400 DB_5mm RT non-spin

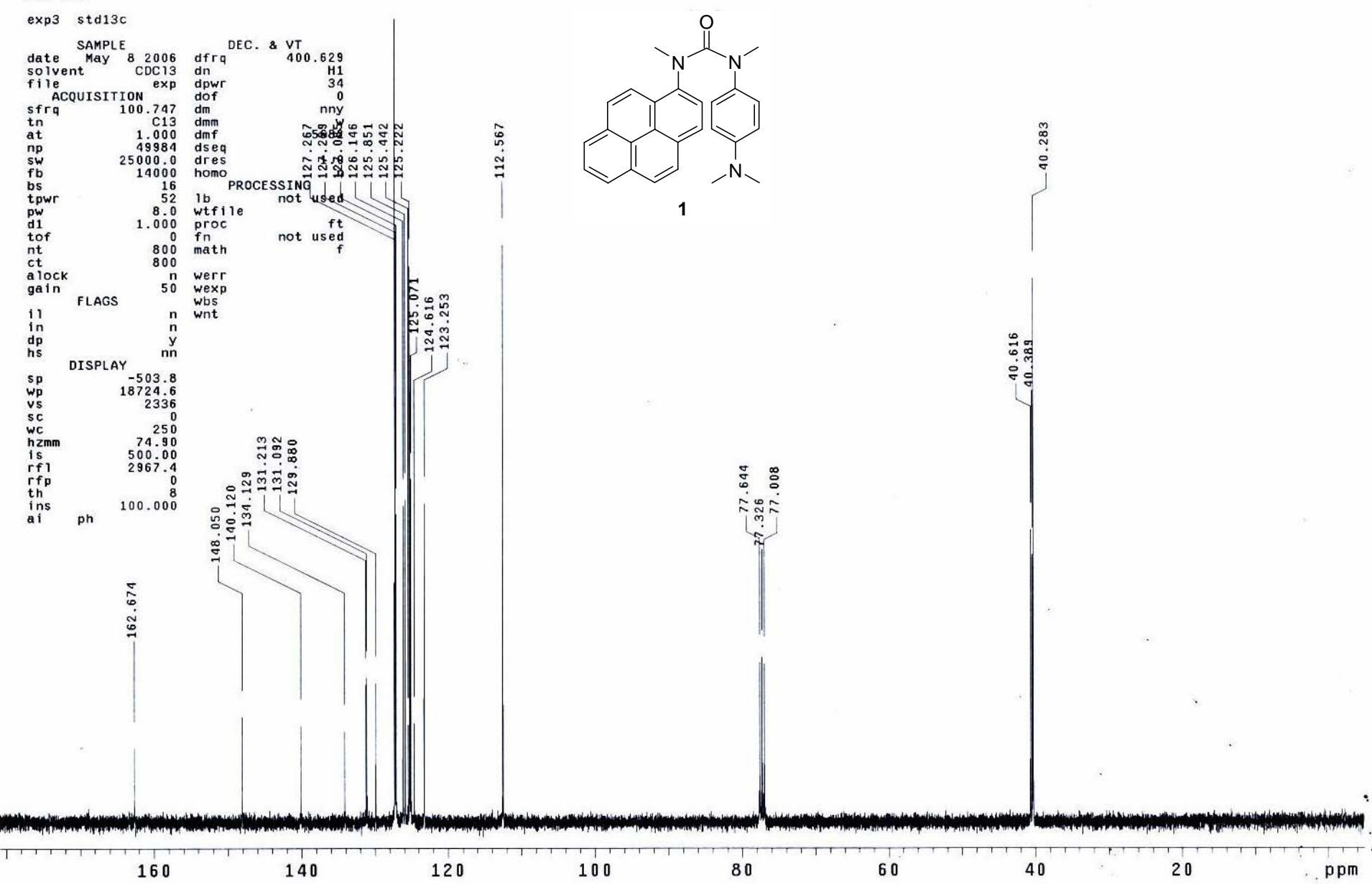


STANDARD $1 \mathrm{H}$ OBSERVE

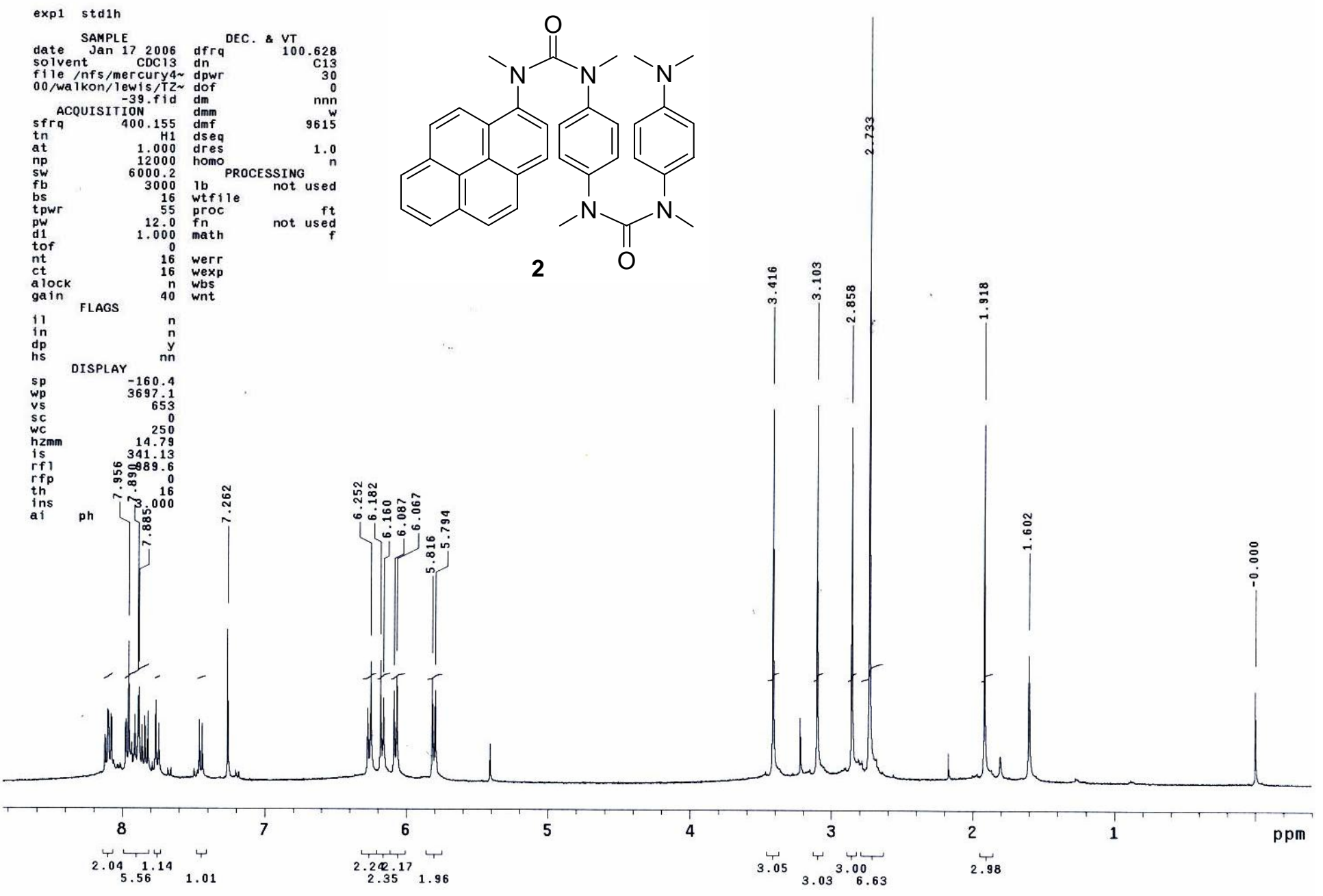




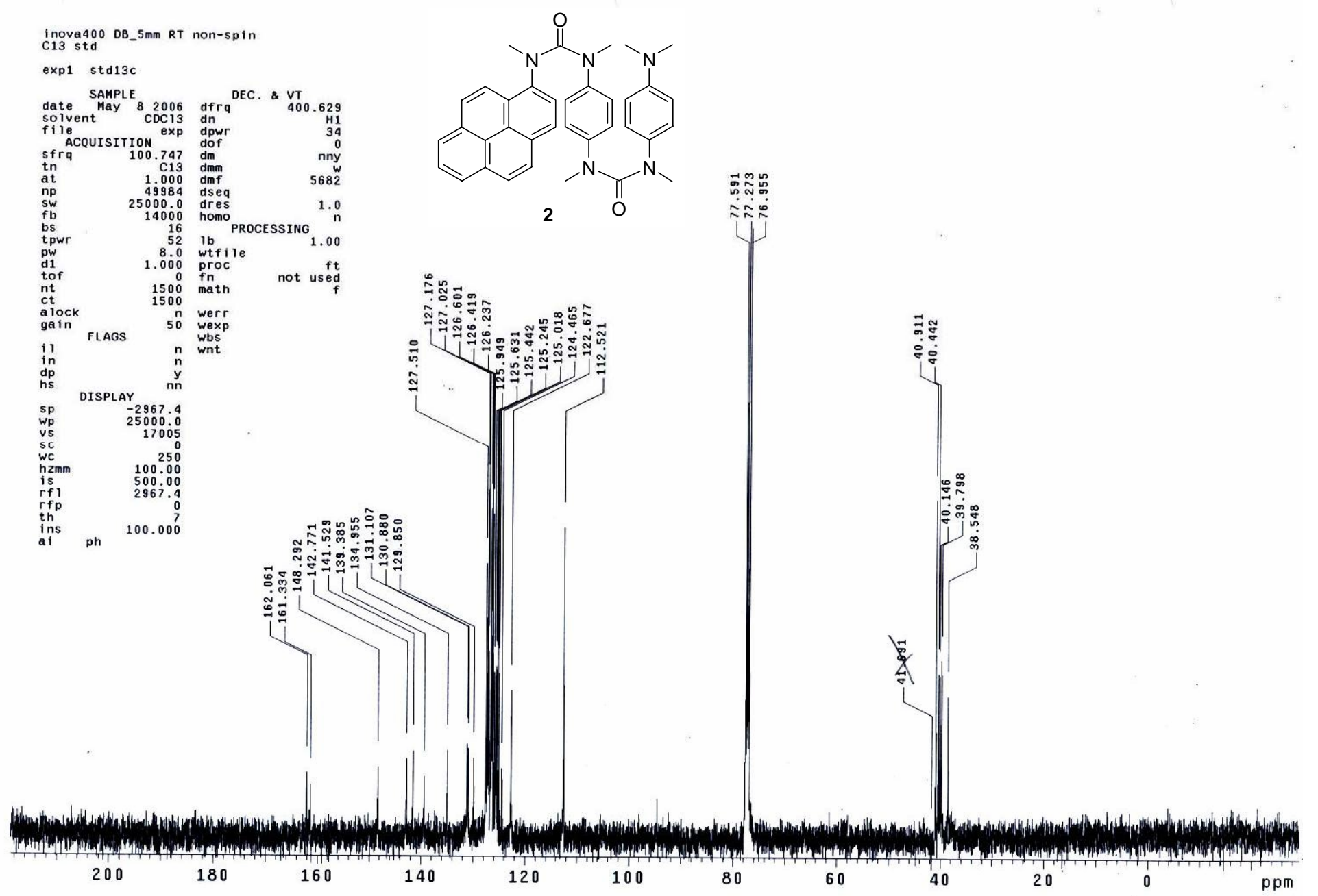




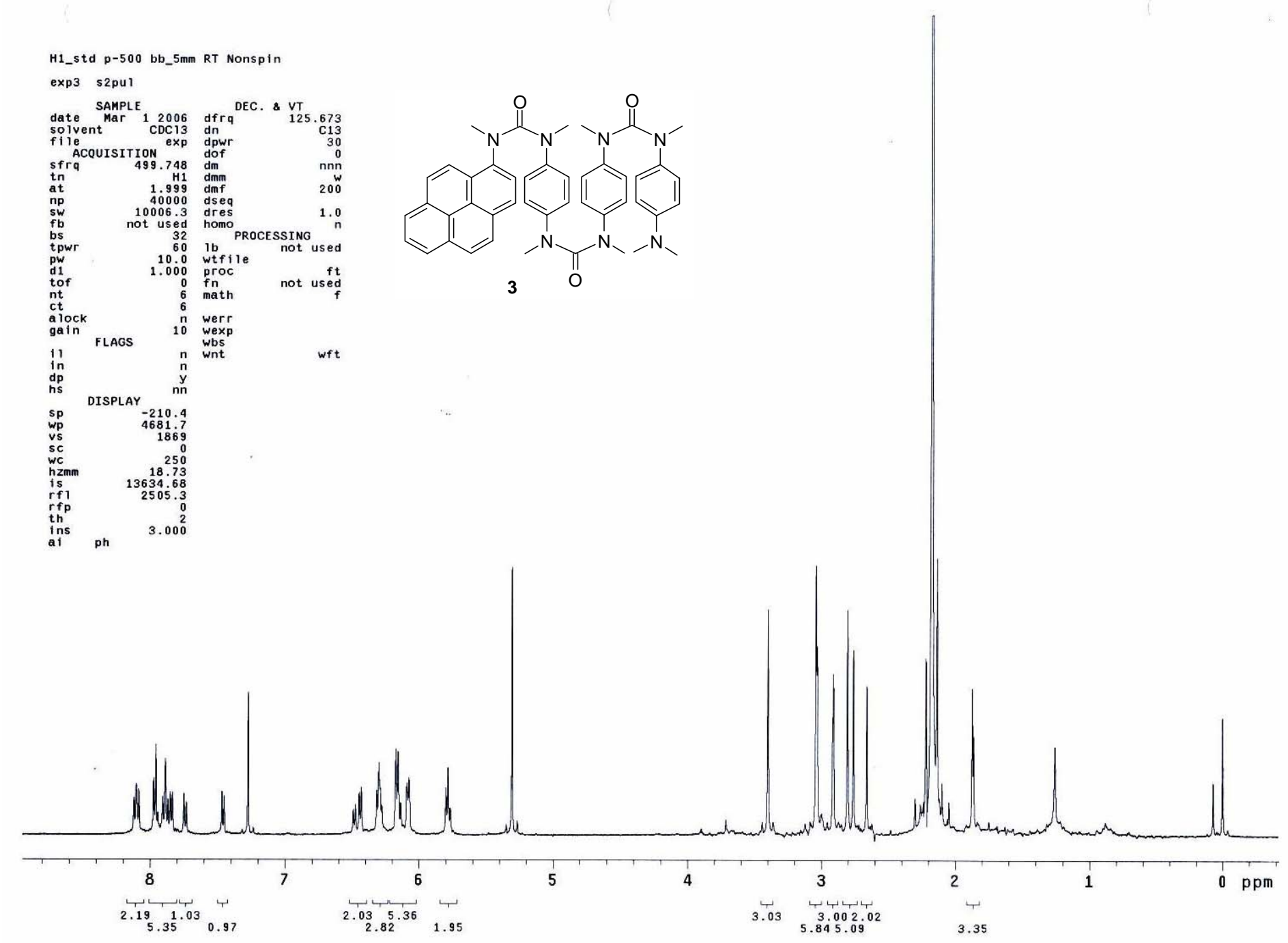




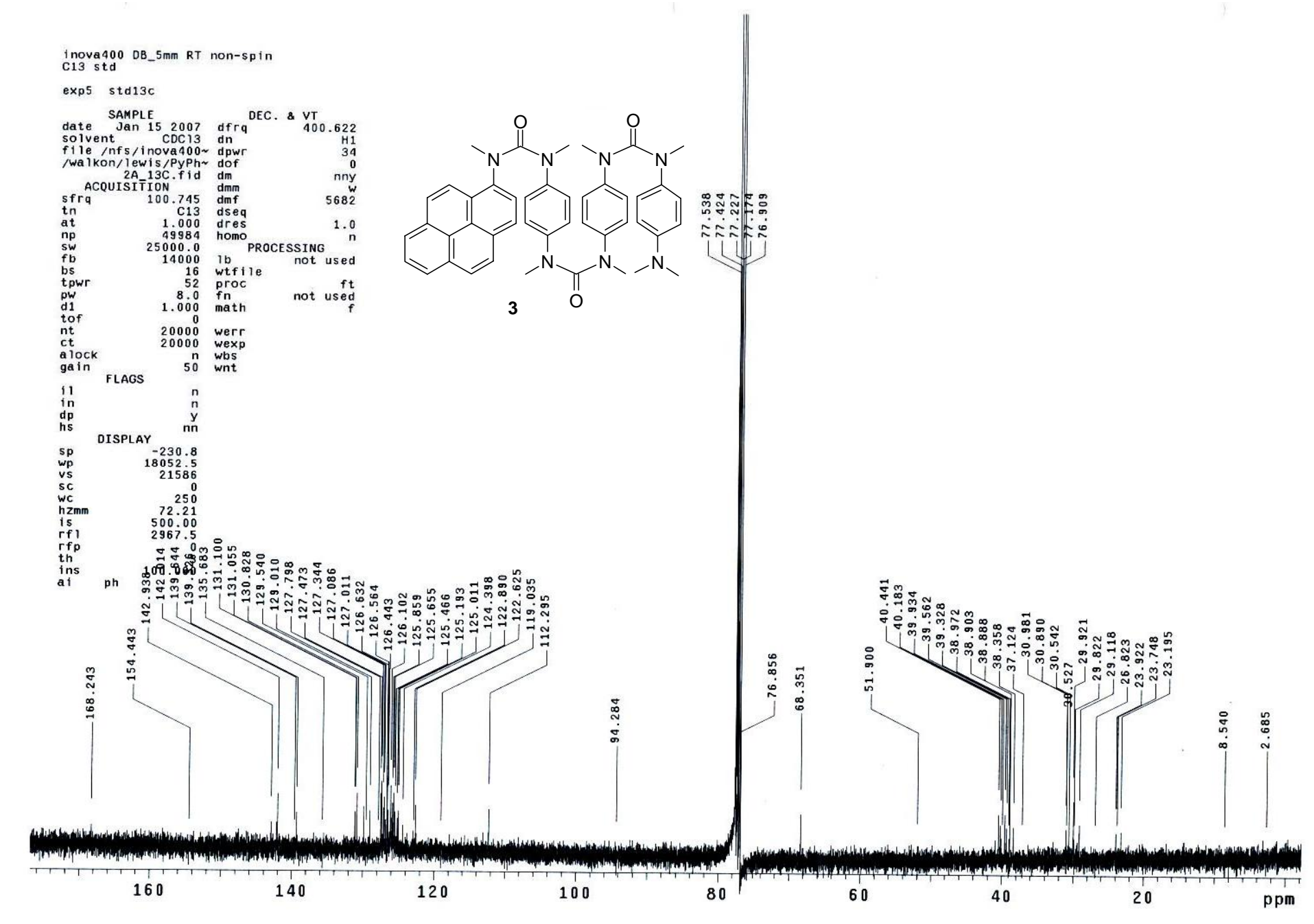


H1_std p-500 bb_5mm RT Nonspin

exp1 s2pul

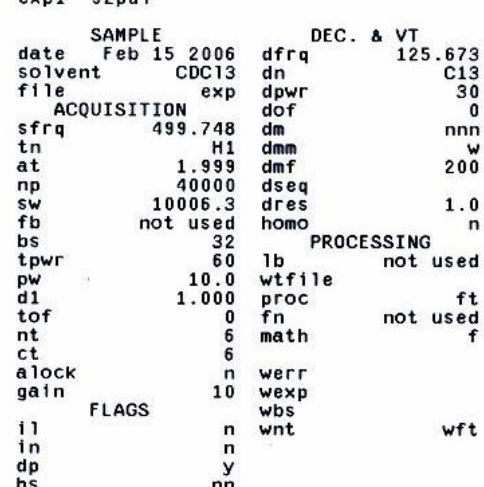

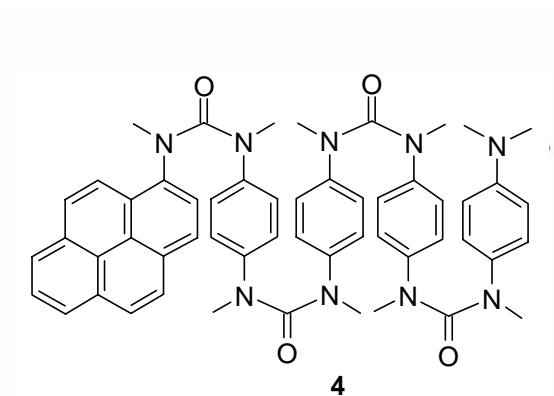

hs DISPLAY nn

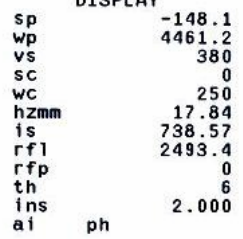

$$
-148.1
$$$$
\begin{array}{r}
250 \\
17.84 \\
\hline 38.57
\end{array}
$$$$
\begin{array}{r}
0 \\
0 \\
6
\end{array}
$$
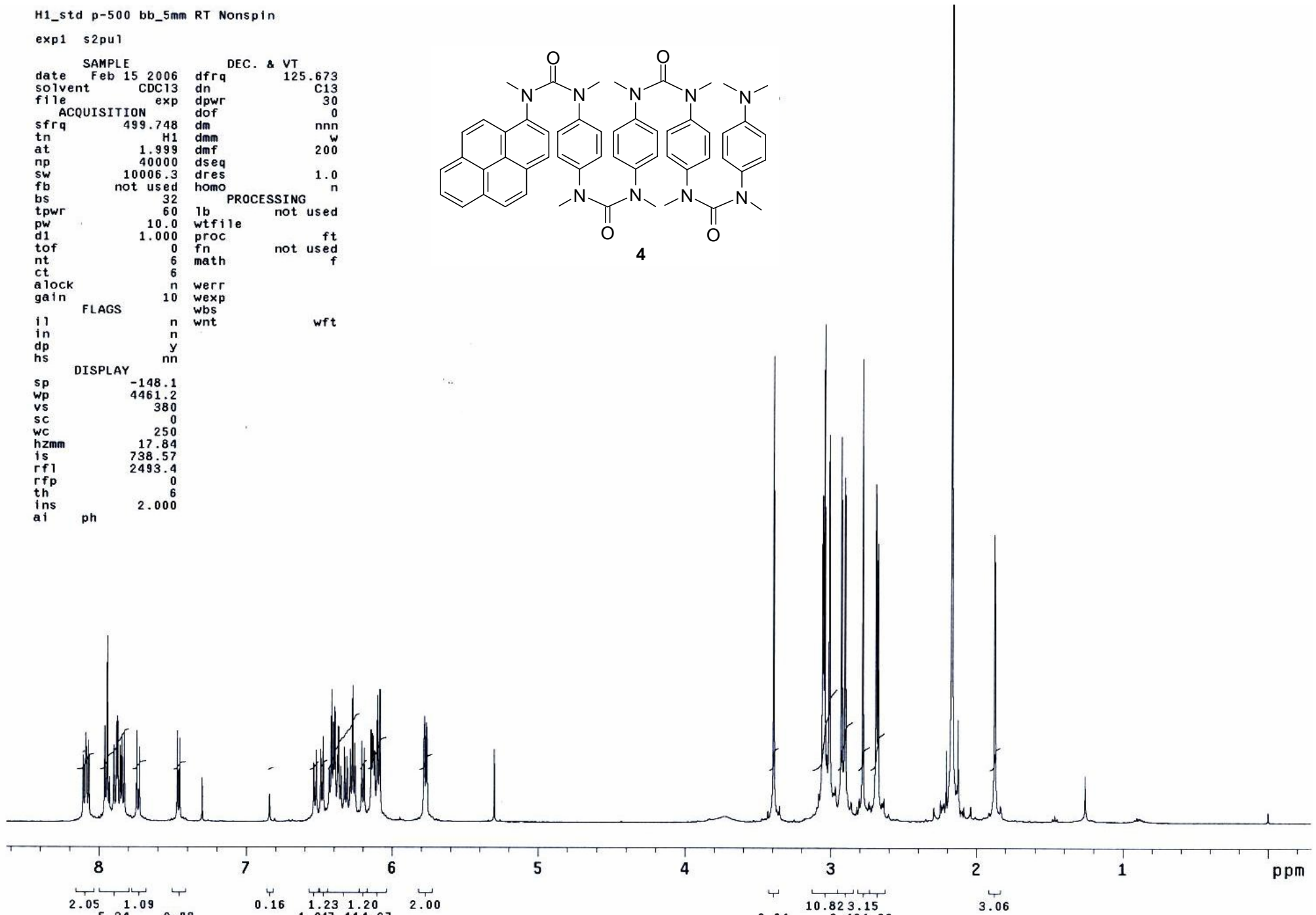


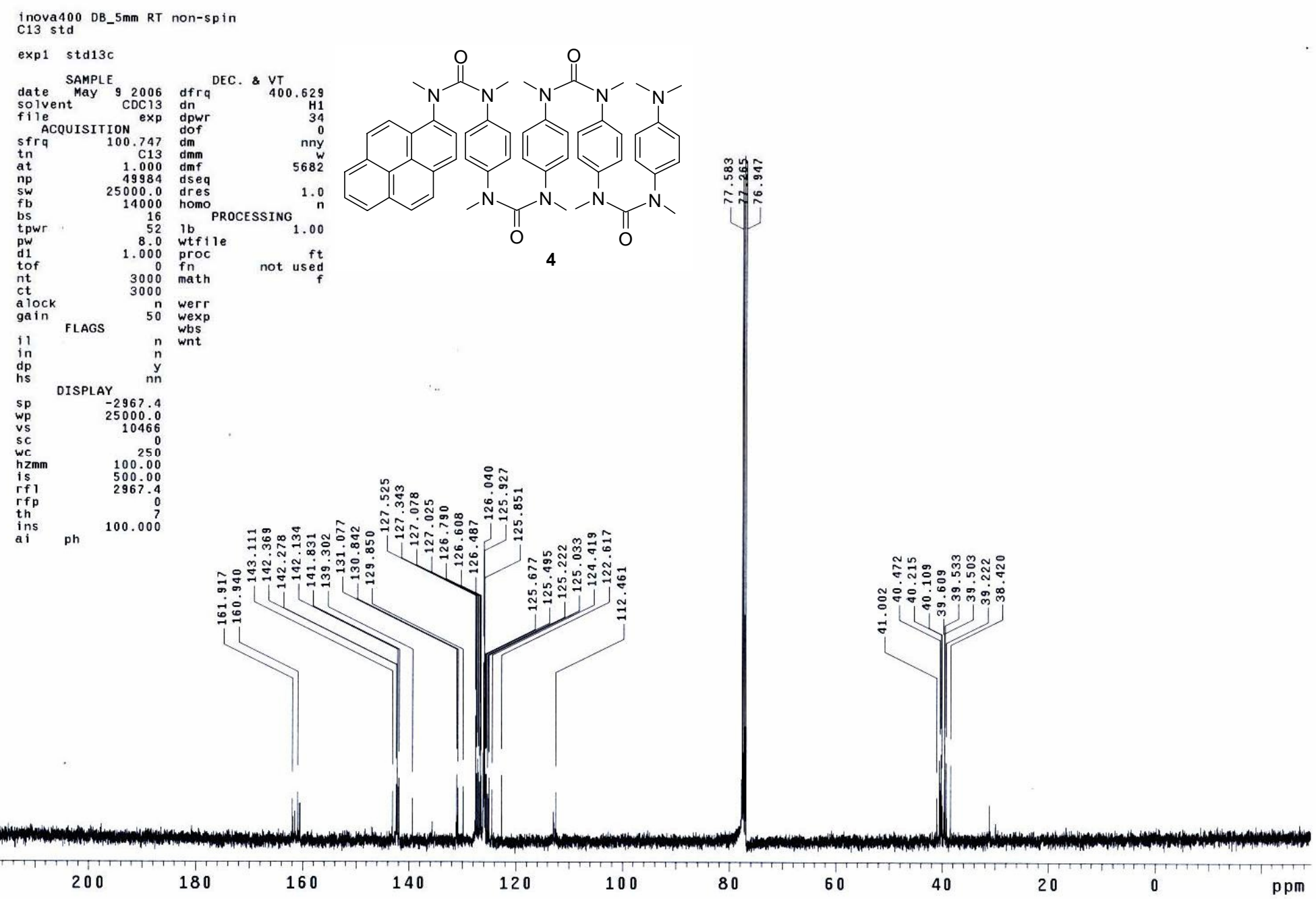




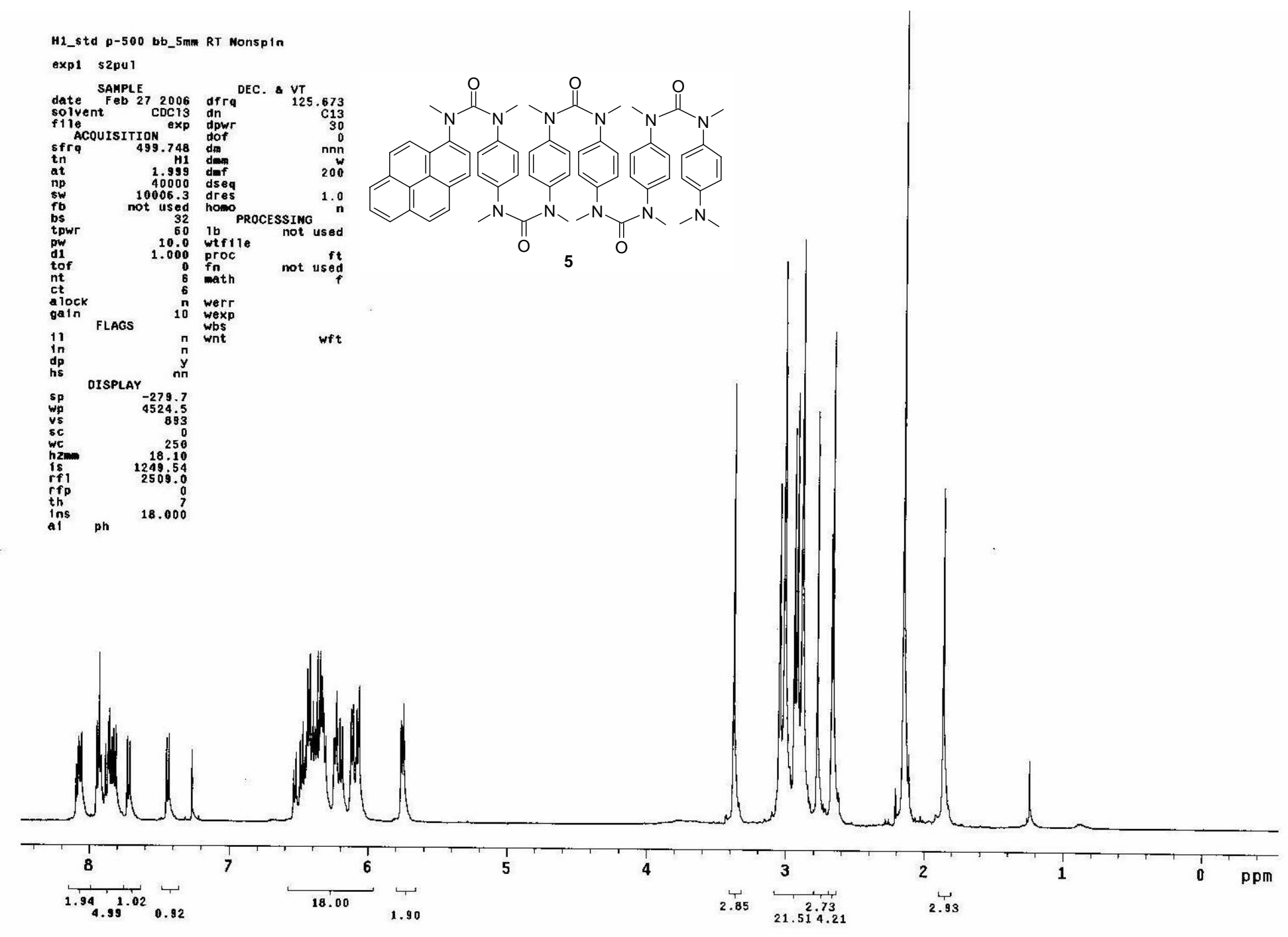




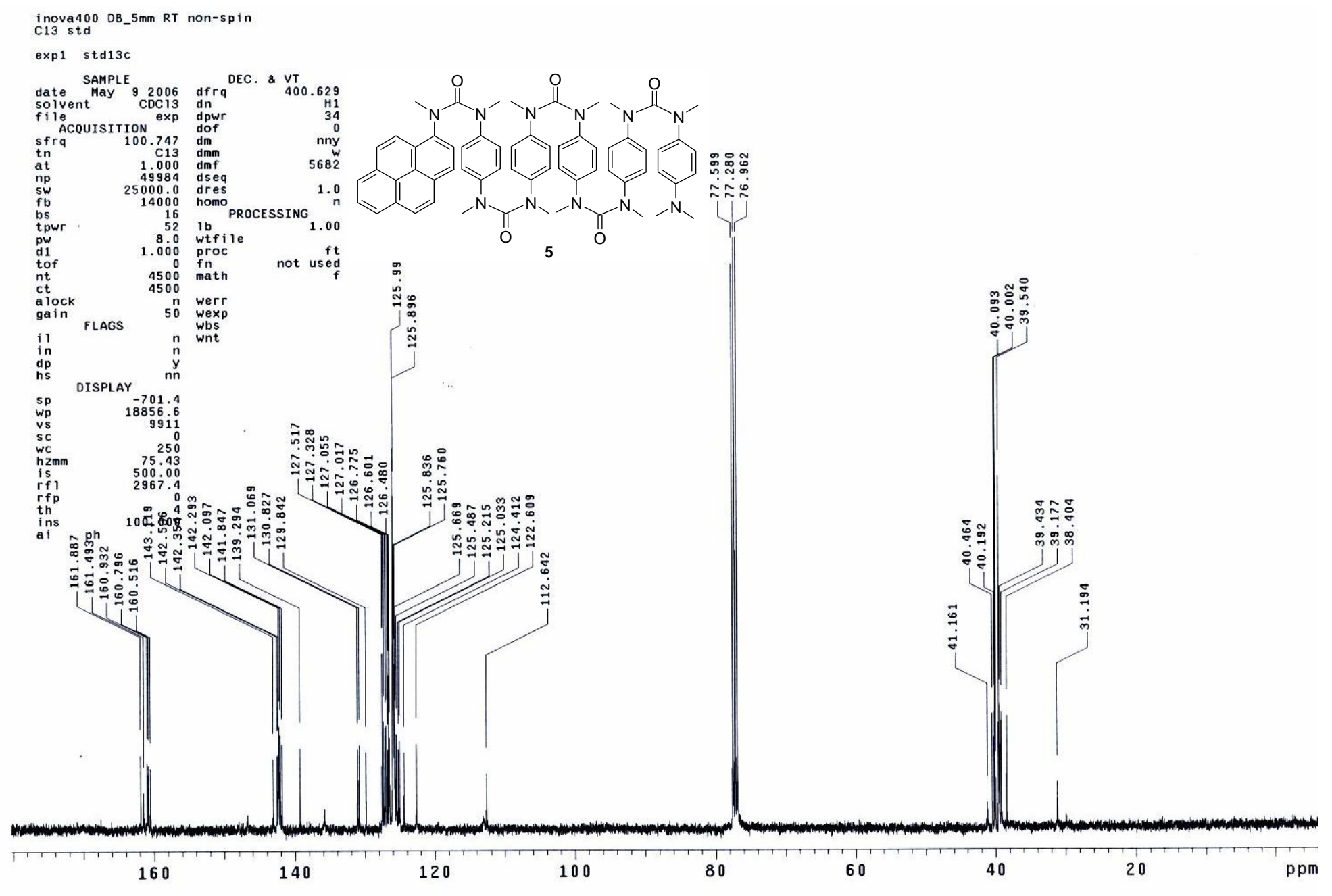




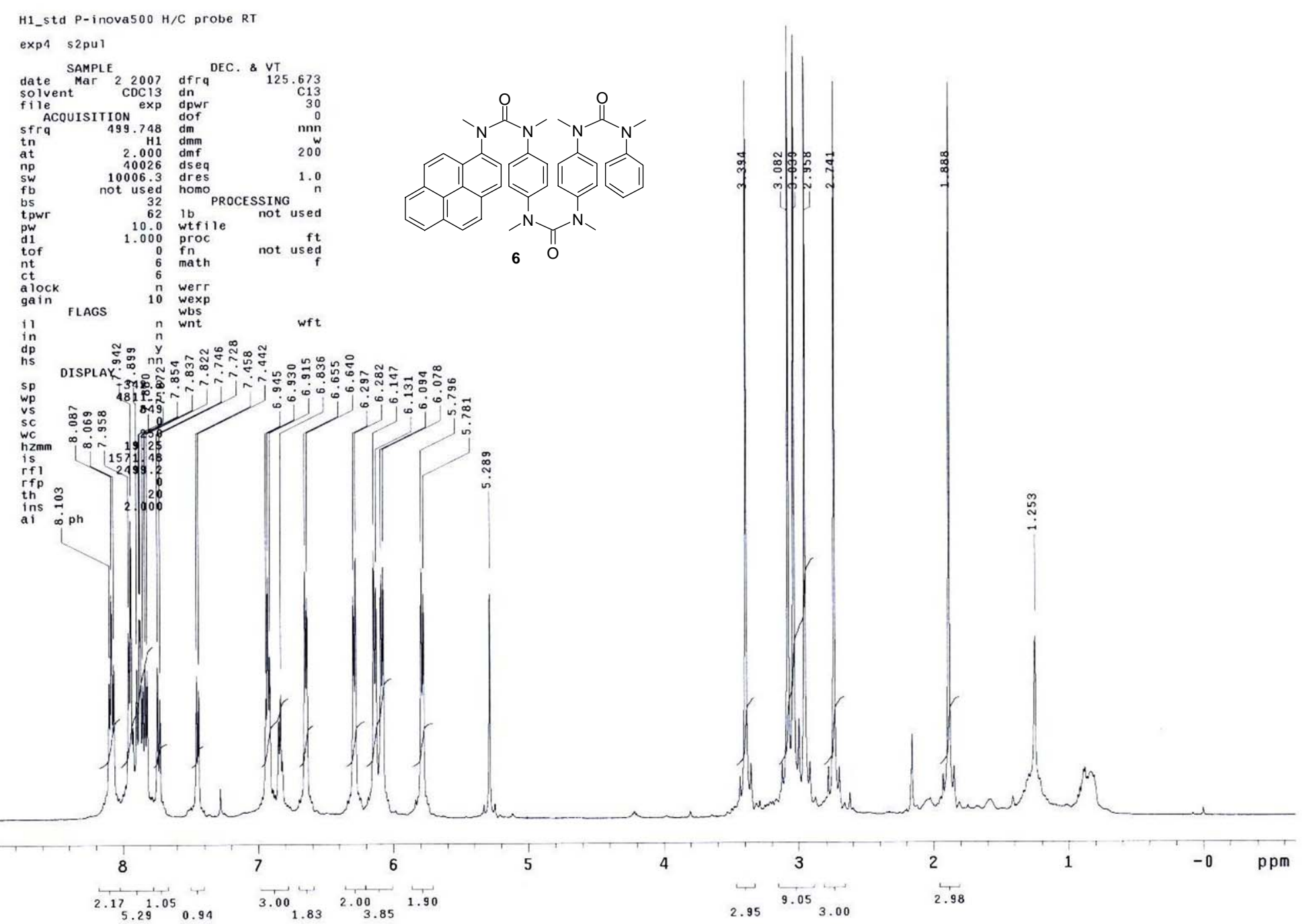


C13 std p-500 H/C probe RT

exp4 s2pul

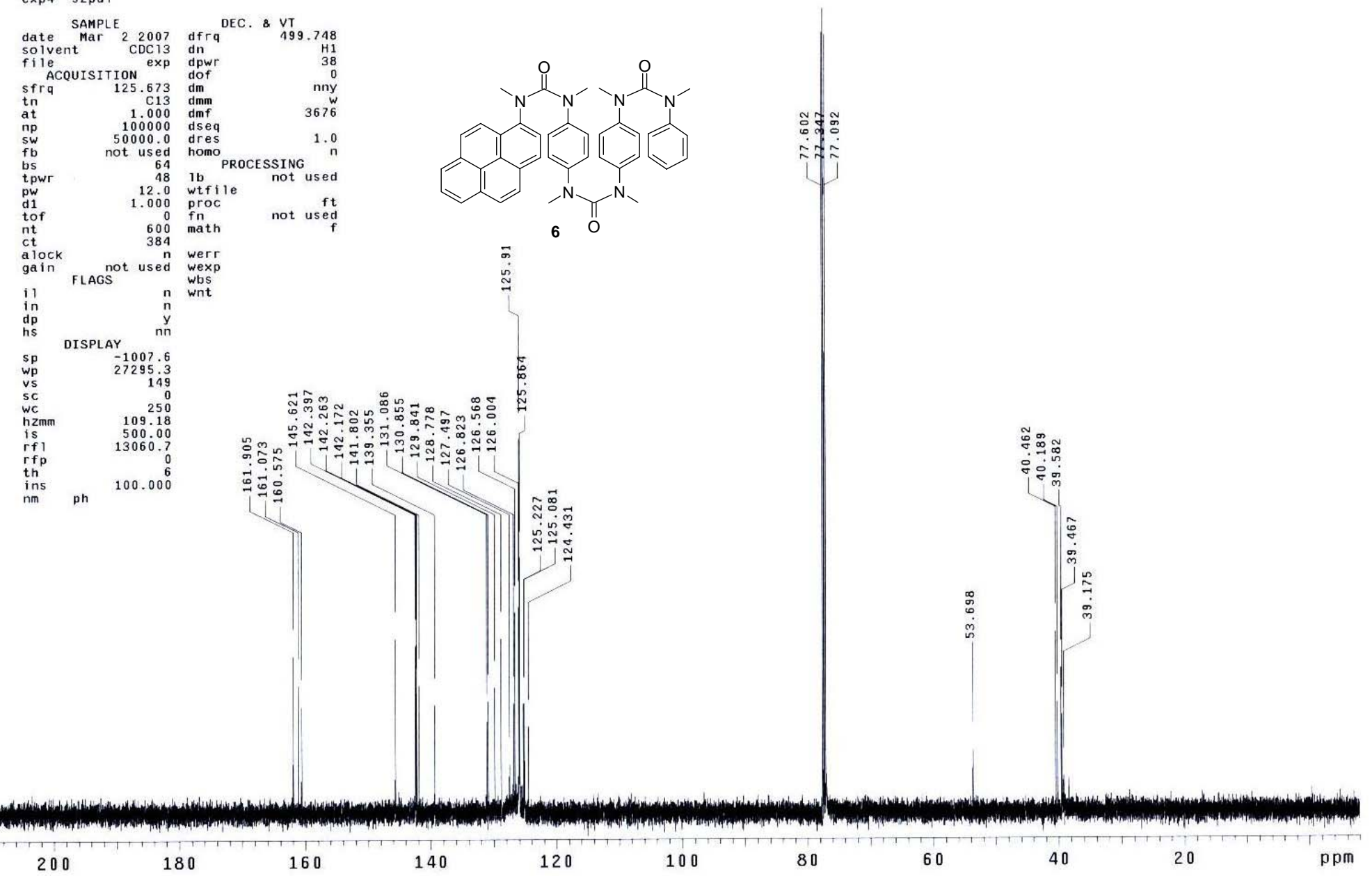




\section{References}

1. Lewis, F. D.; Kurth, T. L.; Delos Santos, G. B. J.Phys. Chem. B 2005, 109, 48934899.

2. CAChe, Release 6.1.10; Fujitsu Ltd.: Miahama-Ku, Chiba City, Chiba, Japan, 2000-2003.

3. Origin, version 6.1; OriginLab Corp.: Northampton, MA, 2000.

4. Raytchev, M.; Pandurski, E.; Buchvarov, I.; Modrakowski, C.; Fiebig, T. J. Phys. Chem. A 2003, 107, 4592-4600. 